\title{
Classification of the FRW universe with a cosmological constant and a perfect fluid of the equation of state $p=w \rho$
}

\author{
Te Ha.* Yongqing Huang $\oplus$ Qianyu Ma $\$$ Kristen D. Pechan $\$$ Timothy \\ J. Renner $₫$ Zhenbin Wu** G. A. Benesh, $+\dagger$ and Anzhong Wang $\$$ \\ Physics Department, Baylor University, Waco, TX 76798-7316
}

(Dated: November 3, 2018)

\begin{abstract}
We systematically study the evolution of the Friedmann-Robertson-Walker (FRW) universe coupled with a cosmological constant $\Lambda$ and a perfect fluid that has the equation of state $p=w \rho$, where $p$ and $\rho$ denote, respectively, the pressure and energy density of the fluid, and $w$ is an arbitrary real constant. Depending on the specific values of $w, \Lambda$, and the curvature $k$ of 3 -dimensional space, we separate all of the solutions into various cases. In each case the main properties of the evolution are given in detail, including the periods of deceleration and/or acceleration, and the existence of big bang, big crunch, and big rip singularities. In some cases, errors in classification and interpretation appearing in standard textbooks have been corrected.
\end{abstract}

PACS numbers: 98.80.-k; 98.80.Jk;04.20.-q; 04.20.Jb

\footnotetext{
*Electronic address: Ha:Ha@baylor.edu

${ }^{\dagger}$ Electronic address: Yongqing·Huang@baylor.edu

${ }^{\ddagger}$ Electronic address: Qianyu’Ma@baylor.edu

$\S$ Electronic address: Kristen'Pechan@baylor.edu

๑Electronic address: Timothy'Renner@baylor.edu

** Electronic address: Zhenbin'Wu@baylor.edu

${ }^{\dagger}$ Electronic address: Greg·Benesh@baylor.edu

${ }_{\ddagger}$ Electronic address: anzhong wang@baylor.edu
} 


\section{INTRODUCTION}

Recent observations of type Ia supernovas (SN) reveal the striking discovery that our universe has lately been in an accelerated expansion phase [1]. Cross checks from cosmic microwave background radiation and large scale structure all confirm these observations [2]. Such an expansion was predicted neither by the standard model of particle physics nor by the standard model of cosmology, and the underlying physics still remains a complete mystery [3]. Since the precise nature and origin of the acceleration have profound implications, understanding them is one of the major challenges of modern cosmology. As the Dark Energy Task Force (DETF) stated [4]: "Most experts believe that nothing short of a revolution in our understanding of fundamental physics will be required to achieve a full understanding of the cosmic acceleration."

Within the framework of general relativity (GR), to account for such an acceleration requires the introduction of either a tiny positive cosmological constant or an exotic component of matter having a very large negative pressure and interacting weakly with other components of matter. This invisible component is usually referred to as dark energy. For a perfect fluid with the equation of state $w=p / \rho$, this implies $w<-1 / 3$, where $p$ and $\rho$ denote, respectively, the pressure and energy density of the fluid. On the other hand, a tiny positive cosmological constant is entirely consistent with all observations carried out thus far [5]. However, when the physical origin of the cosmological constant is considered, several problems are encountered. First, the theoretical expectation values exceed observational limits by 120 orders of magnitude [7]. Second, the corresponding energy density has only recently become comparable with that of matter-otherwise, galaxies would not have formed. Since the energy density of matter is time-dependent, one must explain why only now the two are of the same order. Third, once the cosmological constant begins to dominate the evolution of the universe, it dominates forever. An eternally accelerating universe seems inconsistent with string/M-Theory because it is endowed with a cosmological event horizon that prevents the construction of a conventional S-matrix describing particle interactions [8]. Other problems associated with an asymptotic de Sitter universe in the future were explored in [9].

In view of thse problems, dramatically different models have been proposed, including quintessence [10], DGP branes [11], and the $f(R)$ models [12]. For details, see [3] and 
references therein. However, it is fair to say that so far no convincing model has been proposed.

In this paper, our purpose is two-fold: (i) to provide a complete classification of the FRW models in terms of classical mechanics; and (ii) to correct some errors appearing in standard textbooks. The main idea is simply to cast the dynamical equation in the form of the conservation of total energy of a classical particle with mass $m$ moving under the influence of a potential $V(x)$ [13, 14],

$$
\frac{1}{2} m \dot{x}^{2}+V(x)=E
$$

where $\dot{x} \equiv d x / d t$ and $E$ is the total energy of the system. Taking the derivative of the above equation with respect to $t$, we find that

$$
m \ddot{x}=-\frac{d V(x)}{d x} .
$$

Thus, once the potential $V(x)$ is known in terms of $x$, it can immediately be determined whether the particle is accelerating or decelerating, without it being necessary to integrate Eq.(1.1) explicitly. In addition, it is simple to determine the range of $x$ allowed by the motion. Therefore, if the evolution of the universe problem can be expressed in the above form, we may use the methods of classical mechanics to classify and study all possible solutions.

The rest of the paper is organized as follows: In Sec. II, we consider the Friedmann equation coupled with a cosmological constant and a perfect fluid with the equation of state $p=w \rho$ for any given curvature $k$. After writing it in the form of Eq.(1.1), we study the potential $V(x)$ case by case, and deduce the main properties of each model of the universe. In Sec. III, we present our main conclusions.

It should be noted that classification of (non-relativistic) matter coupled with dark energy was considered recently in [15], and the corresponding Penrose diagrams were also presented. Similar considerations were also carried out in a series of papers, and particular attention was paid to obtaining an effective potential $V(a)$ by fitting observational data sets [14]. In this paper, we shall not consider the fitting of these models, but simply provide a complete classification of all such models. Certainly, some of these models will not be consistent with observations.

In addition, the method used here is not only applicable to general relativity, but also applicable to any theory of gravity, in which the evolution of the universe can be expressed 
in the form (1.1). This certainly includes dark energy models where the equation of state is a function of the scale factor, $w=p / \rho=w(a)$.

In this paper, we use notations and conventions defined in [16].

\section{CLASSIFICATION OF THE FRW UNIVERSE}

The FRW universe is described by the metric [16],

$$
d s^{2}=d t^{2}-a^{2}(t)\left(\frac{d r^{2}}{1-k r^{2}}+r^{2}\left(d \theta^{2}+\sin ^{2} \theta d \phi^{2}\right)\right)
$$

in the spherically symmetric coordinates $x^{\nu}=\{t, r, \theta, \phi\},(\nu=0,1,2,3)$, where $k$ denotes the curvature of the three-dimensional space of constant $t$, which can be set to $k=0, \pm 1$, without loss of generality. $a(t)$ is the expansion factor of the universe. It should be noted that Eq. (2.1) is invariant under the translation,

$$
t^{\prime}=t-t_{s}
$$

where $t_{s}$ is a constant. In the following we shall use this gauge freedom to fix the origin of the timelike coordinate $t$. The expansion factor $a(t)$ of the universe is determined through the Einstein field equations

$$
R_{\mu \nu}-\frac{1}{2} R g_{\mu \nu}=\kappa^{2} T_{\mu \nu}+\Lambda g_{\mu \nu}
$$

where $\kappa^{2}\left[\equiv 8 \pi G / c^{4}\right]$ is the Einstein coupling constant, $\Lambda$ denotes the cosmological constant, and $T_{\mu \nu}$ the energy-momentum tensor of the matter field(s) filling in the universe. For a perfect fluid, we have

$$
T_{\mu \nu}=(\rho+p) u_{\mu} u_{\nu}-p g_{\mu \nu}
$$

where $u_{\mu}$ denotes the four-velocity of the fluid and is given by $u_{\mu}=\delta_{\mu}^{0}$ in the frame of Eq. (2.1). It can be shown [16] that the Einstein field equations (2.3) for the metric (2.1) and energy-momentum tensor (2.4), have only two independent components, which can be cast in the form,

$$
\begin{aligned}
H^{2} & =\frac{8 \pi G}{3} \rho+\frac{1}{3} \Lambda-\frac{k}{a^{2}}, \\
\frac{\ddot{a}}{a} & =-\frac{4 \pi G}{3}(\rho+3 p)+\frac{1}{3} \Lambda,
\end{aligned}
$$


where $H \equiv \dot{a} / a$. Note that in writing the above equation, we have chosen units such that the speed of light is one. On the other hand, the conservation law of matter fields, $\nabla^{\nu} T_{\mu \nu}=0$, yields

$$
\dot{\rho}+3 H(\rho+p)=0 .
$$

It can be shown that this equation is not independent, and can be obtained from Eqs.(2.5) and (2.6).

Note that we have three unknowns, $a, \rho$ and $p$, but only two independent equations. Thus, to close the system, one more equation is required. Usually this is given by the equation of state of the matter field. In this paper, we consider cases in which

$$
p=w \rho,
$$

where $w$ is an arbitrary real constant. When $w \geq-1 / 3$ and $\rho>0$, the fluid satisfies all the energy conditions, weak, strong and dominant [17], and usually is referred to as "normal" matter. In particular, the fluid with $w=0$ is a dust, and often referred to as matter [18]. When $w<-1 / 3$, the fluid does not satisfy strong energy condition, and has a positive contribution to the acceleration of the universe, as can be seen from Eq. (2.6). This kind of fluid is usually referred to as dark energy. Since a cosmological constant corresponds to a perfect fluid with $w=-1$, where the corresponding energy density and pressure are defined by $\rho_{\Lambda}=-p_{\Lambda}=\Lambda /(8 \pi G)$, it is often considered as a particular dark energy. A fluid with $w<-1$ is called phantom, which does not satisfy any of the three energy conditions.

Inserting Eq.(2.8) into Eq.(2.7) and integrating once gives,

$$
\rho=\rho_{0}\left(\frac{a_{0}}{a}\right)^{3(1+w)},
$$

where $\rho_{0}$ and $a_{0}$ are integration constants. Since $\rho_{0}$ represents the energy density when $a=a_{0}$, we assume that it is strictly positive $\left(\rho_{0}>0\right)$. Without loss of generality, we can always set $a_{0}=1$. Then, it can be shown that the Friedmann equation (2.5) can be cast in the form of Eq.(1.1) with $m=1, E=0, x(t)=a(t)$, i.e.,

$$
\frac{1}{2} \dot{a}^{2}+V(a)=0,
$$

where

$$
V(a)=\frac{1}{2} k-\frac{1}{6} \Lambda a^{2}-\frac{\mathcal{C}}{a^{1+3 w}},
$$


with

$$
\mathcal{C} \equiv \frac{4 \pi G \rho_{0}}{3}>0
$$

It should be noted that the equivalency of the Friedmann equation (2.10) with the conservation law of mechanical energy Eq.(1.1) is rather a formality. In particular, energy in GR is not well-defined, while a force is no longer a vector. Therefore, although we can still use these terms to describe the motion of the universe in the following discussions, one should always keep this in mind.

When $w=0$ the problem reduces to the one treated in [16]. To study the problem further, we consider separately the cases $k=0, \pm 1$.

\section{A. $k=0$}

When $k=0$, Eq.(2.11) reduces to

$$
V(a)=-\frac{1}{6} \Lambda a^{2}-\frac{\mathcal{C}}{a^{1+3 w}}
$$

It is convenient to consider the cases $\Lambda>0, \Lambda=0$, and $\Lambda<0$ separately.

1. $\Lambda>0$

When $\Lambda>0$, Eq.(2.13) can be written as

$$
V(a)=-\frac{1}{6} \Lambda a^{2}\left(1+\frac{\tilde{\mathcal{C}}}{a^{3(1+w)}}\right),
$$

where $\tilde{\mathcal{C}} \equiv 6 \mathcal{C} / \Lambda>0$. Thus, in this case $V(a)$ is always non-positive. Depending on the value of $w$, the evolution of the universe can differ significantly. Thus, we further distinguish the following sub-cases:

$$
\begin{aligned}
& \text { (1) } w>-\frac{1}{3}, \quad \text { (2) } w=-\frac{1}{3}, \quad \text { (3) }-1<w<-\frac{1}{3}, \\
& \text { (4) } w=-1, \quad \text { (5) } w<-1 .
\end{aligned}
$$

Case A.1.1) $w>-\frac{1}{3}$ : Then, we find that $V(a) \rightarrow-\infty$ for both $a=0$ and $a \rightarrow \infty$. It also has a maximum at $a=a_{m} \equiv(3(1+3 w) \mathcal{C} / \Lambda)^{1 /(3(1+w))}$, for which

$$
\ddot{a}= \begin{cases}<0, & a<a_{m}, \\ =0, & a=a_{m}, \\ >0, & a>a_{m} .\end{cases}
$$




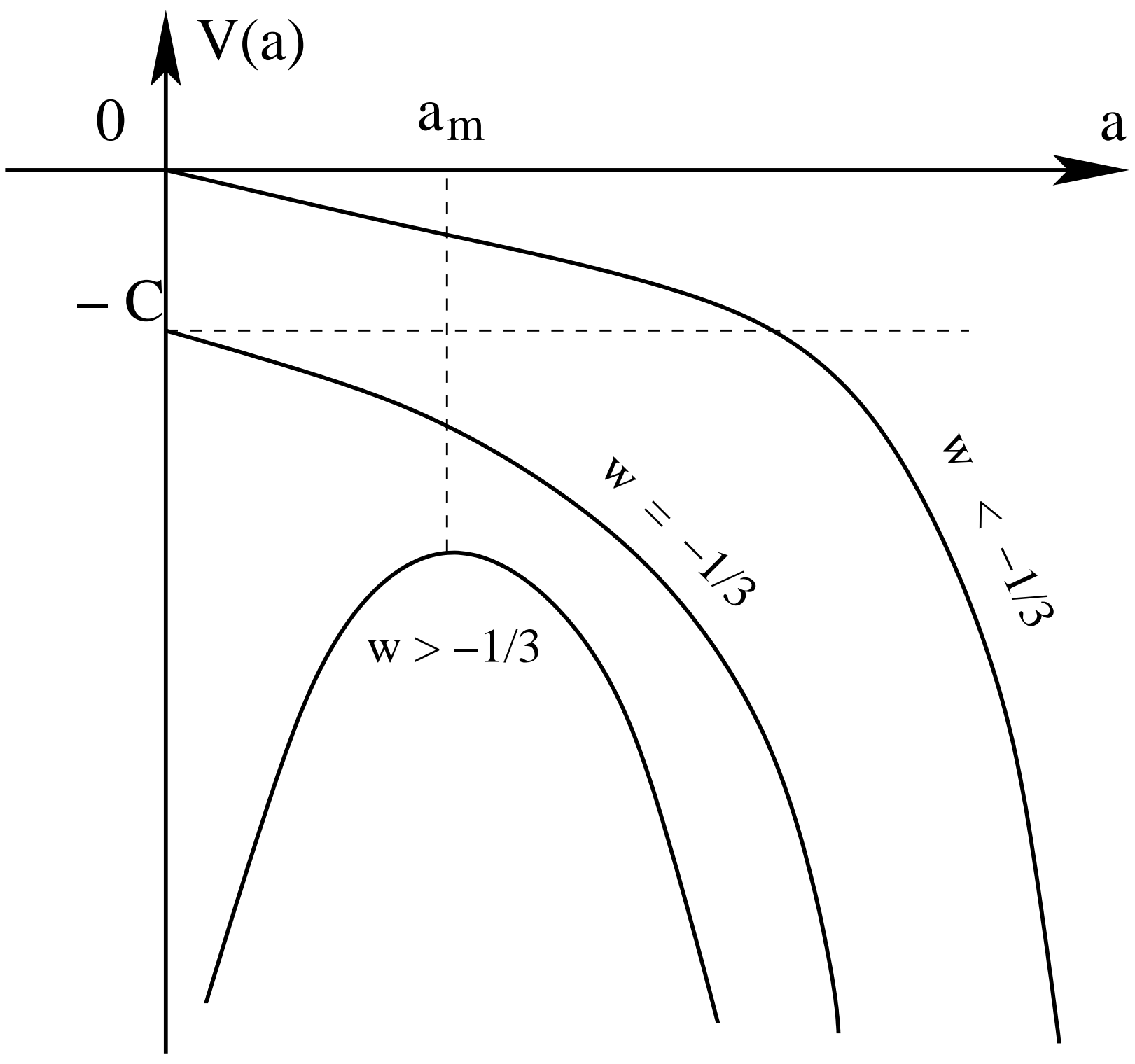

FIG. 1: The potential given by Eq.(2.14) for $k=0$ and $\Lambda>0$.

Fig. 1 schematically shows the potential, represented by the bottom line. Therefore, in this case, the evolution of the universe is dominated by matter in early times and $a(t) \propto t^{2 /[3(1+w)]}$, for which $\ddot{a}<0$. As the universe expands to $a=a_{m}$, it reaches the turning point, after which the expansion begins accelerating, i.e., $\ddot{a}>0$ for $a>a_{m}$. The universe is asymptotically de Sitter, $a(t) \propto e^{\sqrt{\Lambda / 3} t}$.

Case A.1.2) $w=-\frac{1}{3}$ : In this case, we find

$$
V(a)=-\frac{1}{6} \Lambda a^{2}-\mathcal{C}
$$




$$
\begin{gathered}
= \begin{cases}-\mathcal{C}, & a=0, \\
-\infty, & a \rightarrow \infty,\end{cases} \\
\ddot{a}=-\frac{d V(a)}{d a}=\frac{1}{3} \Lambda a \geq 0 .
\end{gathered}
$$

The middle line of Fig. 1 represents the potential for this case. The universe is always accelerating after the initial moment when $a=0$. On the other hand, from Eq.(2.9) we find that $\rho \propto a^{-2} \rightarrow \infty$ as $a \rightarrow 0$; that is, a big bang singularity still occurs at $a=0$.

Case A.1.3) $-1<w<-\frac{1}{3}$ : In this case, the potential is represented by the upper line in Fig. 11, and

$$
\ddot{a}=-\frac{d V(a)}{d a}=\frac{1}{3} \Lambda a+(3|w|-1) \mathcal{C} a^{3|w|-2} \geq 0,
$$

where the equality holds only at $a=0$ for $w \neq-2 / 3$. Thus, in this case the universe is always accelerating. Note that in the present case $a=0$ still represents a big bang singularity, as can be seen from Eq.(2.9). It is also interesting to note that at $a=0$, we have $\dot{a}=\ddot{a}=0$, except for $w=-2 / 3$. Thus, when $w \neq-2 / 3$ the point $a=0$ is a stationary point. However, it is not stable, and any perturbation will cause the universe to expand. When $w=-2 / 3$, we have $\dot{a}(a=0)=0$ and $\ddot{a}(a=0)=(3|w|-1) \mathcal{C}>0$. Therefore, $a=0$ is not stationary in the latter case, and the positive force causes the universe to expand automatically.

Case A.1.4) $w=-1$ : In this case, matter acts as a vacuum energy, and the potential is given by

$$
V(a)=-\frac{1}{6} \Lambda_{e f f} a^{2}
$$

where $\Lambda_{\text {eff }} \equiv \Lambda+6 \mathcal{C}$. Therefore, the universe is de Sitter, and

$$
a(t)=e^{\sqrt{\Lambda_{e f f} / 3}\left(t-t_{0}\right)} .
$$

Recall that the de Sitter space is free of any kind spacetime singularities at $a=0$, as well as at $a=\infty[16]$.

Case A.1.5) $w<-1$ : In this case, the behavior of the potential $V(a)$ and $a(t)$ are similar to the case $-1<w<-1 / 3$, except that now $\rho \propto a^{3(|w|-1)}$ is not singular at $a=0$. However, there is a singularity at $a=\infty$, which is usually referred to as a big rip singularity. At $a=0$ we have $\dot{a}=\ddot{a}=0$; thus, this point also represents an unstable stationary point.

Finally, we note that for all cases with $k=0$, the corresponding Friedmann equation can be integrated explicitly, and the corresponding solutions are given by,

$$
a(t)=\left\{\left(\frac{6 \mathcal{C}}{\Lambda}\right)^{1 / 2} \sinh \left[(1+w) \sqrt{\frac{3 \Lambda}{4}}\left(t-t_{s}\right)\right]\right\}^{\frac{2}{3(1+w)}},
$$


for $w \neq-1$, where $t_{s}$ is given by

$$
t_{s}=t_{0}-\frac{1}{1+w} \sqrt{\frac{4}{3 \Lambda}} \sinh ^{-1}\left(\sqrt{\frac{\Lambda}{6 \mathcal{C}}}\right)
$$

so that $a\left(t=t_{0}\right)=1$. For $w>-1$, without loss of generality, we use the gauge freedom of Eq.(2.2) to set $t_{s}=0$, so that the big bang singularity occurs at $t=0$. This will be the case for the rest of this paper.

When $w=-1$, the solution is de Sitter, given by Eq.(2.20), which is free of all spacetime singularities. The solution is valid for all $t$, that is, $t \in(-\infty, \infty)$. For the cases in which $w<-1$, there is no translation, so that the big rip singularity occurs exactly at $t=t_{s}$. Note that when $w<-1$, solutions of Eq.(2.21) are valid only when $t \in\left(-\infty, t_{s}\right)$, for which

$$
\begin{aligned}
a(t) & =\left\{\sqrt{\frac{6 \mathcal{C}}{\Lambda}} \sinh \left[\sqrt{\frac{3 \Lambda}{4}}(|w|-1)\left(t_{s}-t\right)\right]\right\}^{-\frac{2}{3(|w|-1)}} \\
& = \begin{cases}\infty, & t=t_{s} \\
1, & t=t_{0}, \quad(w<-1) \\
0, & t=-\infty\end{cases}
\end{aligned}
$$

Since $\rho \propto a^{3(|w|-1)}$ spacetime is indeed not singular at $a(t=-\infty)=0$, but is singular at $a\left(t=t_{s}\right)=\infty$.

In Fig. 2 we summarize the main properties of the solutions for $k=0$ and $\Lambda>0$ for different values of $w$.

2. $\Lambda=0$

In this case, we have

$$
\begin{aligned}
V(a) & =-\frac{\mathcal{C}}{a^{1+3 w}} \leq 0, \\
\ddot{a} & =-(3 w+1) \frac{\mathcal{C}}{a^{2+3 w}} \\
& = \begin{cases}<0, & w>-1 / 3, \\
=0, & w=-1 / 3, \\
>0, & w<-1 / 3 .\end{cases}
\end{aligned}
$$

Fig. 3 shows the potential $V(a)$. As in the previous case, we may integrate the Friedmann equation (2.5) to obtain explicit solutions for $a(t)$ and $\rho(t)$,

$$
a(t)= \begin{cases}{\left[3(1+w) \sqrt{\frac{\mathcal{C}}{2}}\left(t-t_{s}\right)\right]^{\frac{2}{3(1+w)}},} & w \neq-1 \\ e^{\sqrt{2 \mathcal{C}}\left(t-t_{0}\right)}, & w=-1\end{cases}
$$




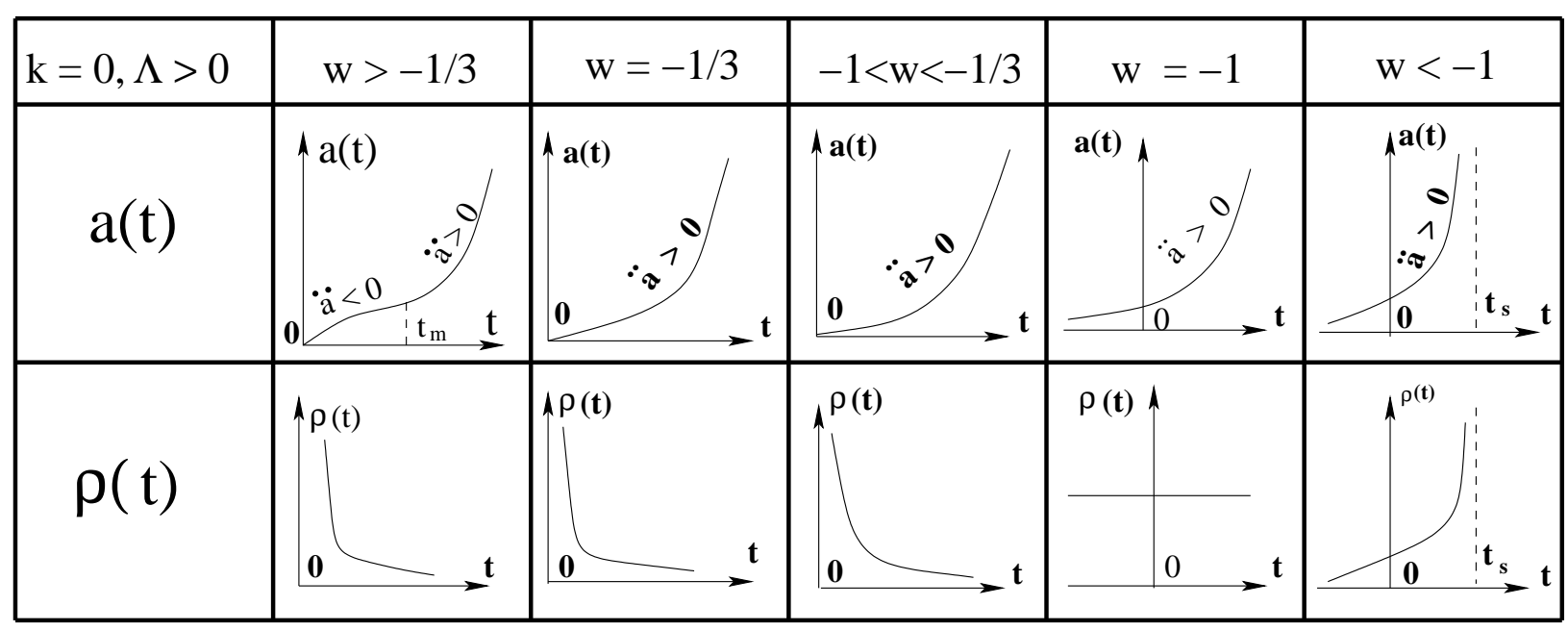

FIG. 2: The expansion factor $a(t)$, the acceleration $\ddot{a}(t)$, and the energy density $\rho(t)$ for $k=0$ and $\Lambda>0$. A big bang singularity occurs at $t=0$ for $w>-1$. It is de Sitter for $w=-1$, which is free of any kind of spacetime singularities. When $w<-1$, a big rip singularity occurs at $t=t_{s}$, in which $a\left(t_{s}\right)=\infty=\rho\left(t_{s}\right)$.

$$
\rho(t)=\left\{\begin{array}{ll}
\frac{\tilde{\rho}_{0}}{\left(t-t_{s}\right)^{2}}, & w \neq-1, \\
\rho_{0}, & w=-1,
\end{array},\right.
$$

where $\tilde{\rho}_{0} \equiv 2 \rho_{0} /\left[9(1+w)^{2} \mathcal{C}\right]$, and

$$
t_{s}=t_{0}-\frac{1}{1+w} \sqrt{\frac{2}{9 \mathcal{C}}}
$$

When $w<-1$, Eq.(2.25) shows that $a(t)$ remains real and positive when $t \in\left(-\infty, t_{s}\right)$. As $t \rightarrow-\infty$, both $a(t)$ and $\rho(t)$ vanish; however, when $t \rightarrow t_{s}$ each becomes unbounded-that is, a big rip singularity is developed there.

In Fig. 4 we summarize the main properties of the $k=0$ and $\Lambda=0$ solutions for different values of $w$.

3. $\Lambda<0$

In this case, we have

$$
V(a)=\frac{1}{6}|\Lambda| a^{2}-\frac{\mathcal{C}}{a^{1+3 w}}
$$




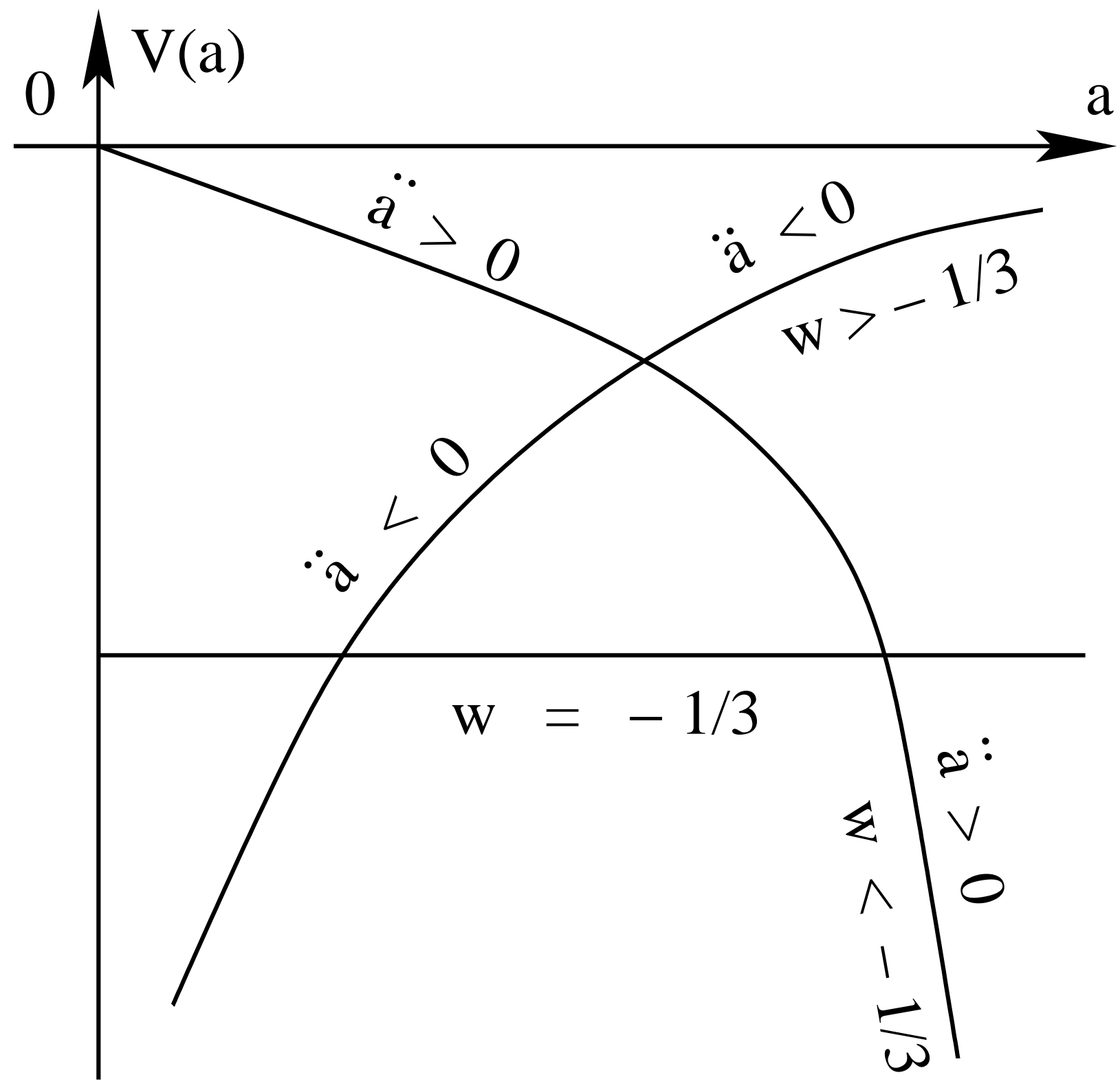

FIG. 3: The potential given by Eq.(2.14) for $k=0$ and $\Lambda=0$.

which is illustrated in Fig. 5 for various values of $w$. It can be shown that the corresponding expansion factor and energy density are given, respectively, by

$$
\begin{aligned}
& a(t)=\left\{\sqrt{\frac{6 \mathcal{C}}{|\Lambda|}} \sin \left[\sqrt{\frac{3|\Lambda|}{4}}(1+w)\left(t-t_{s}\right)\right]\right\}^{\frac{2}{3(1+w)}}, \\
& \rho(t)=\frac{\tilde{\rho}_{0}}{\sin ^{2}\left(\sqrt{\frac{3|\Lambda|}{4}}(1+w)\left(t-t_{s}\right)\right)},
\end{aligned}
$$




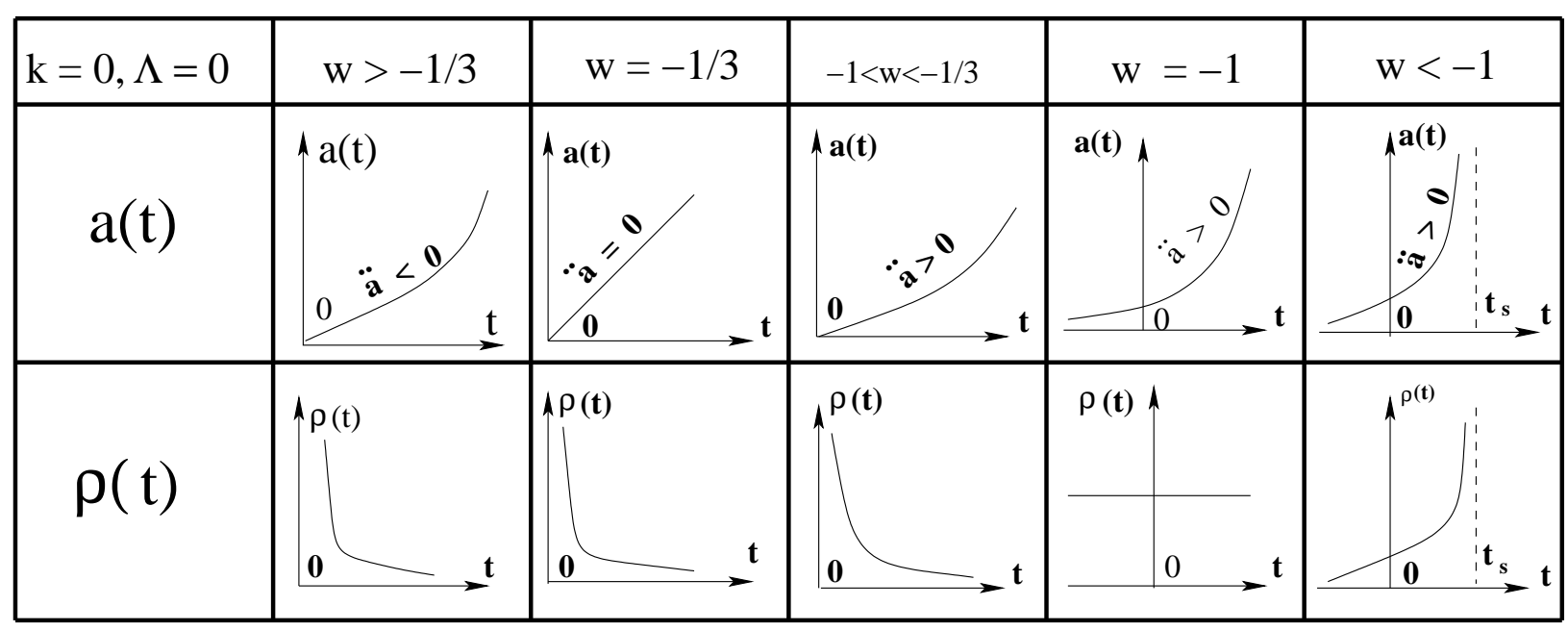

FIG. 4: The expansion factor $a(t)$, the acceleration $\ddot{a}(t)$, and the energy density $\rho(t)$ for $k=0$ and $\Lambda=0$. There is a big bang singularity at $a=0$ for all cases with $w>-1$. The spacetime is de Sitter for $w=-1$. When $w<-1$, a big rip singularity is developed at $t=t_{s}$, at which we have $a\left(t_{s}\right)=\rho\left(t_{s}\right)=\infty$

for $w \neq-1$, and

$$
\begin{aligned}
& a(t)=e^{\sqrt{\Lambda_{e f f} / 3}\left(t-t_{0}\right)}, \\
& \rho(t)=\rho_{0},
\end{aligned}
$$

for $w=-1$, where $\Lambda_{e f f}=|\Lambda|-6 \mathcal{C}>0$. Also,

$$
\begin{aligned}
& t_{s} \equiv t_{0}-\frac{\sqrt{4 /(3|\Lambda|)}}{1+w} \sin ^{-1}\left(\sqrt{\frac{|\Lambda|}{6 \mathcal{C}}}\right), \\
& \tilde{\rho}_{0} \equiv \frac{|\Lambda| \rho_{0}}{6 \mathcal{C}}
\end{aligned}
$$

As in the previous cases, we use gauge freedom (2.2) to set $t_{s}=0$ for $w>-1$, while keeping $t_{s}$ unchanged for $w \leq-1$.

Case A.3.1) $w>-\frac{1}{3}$ : In this case, as shown in Fig. 5, $V\left(a>a_{m}\right)>0$; so, the motion for $a>a_{m}$ is forbidden. If the universe starts expanding from the big bang when $a=0$, after expanding to its maximal radius $a_{m}$ it will start collapsing until a big crunch singularity is developed at $t=2 t_{m}$. This is illustrated in Figs. [5] and [6, where $a_{m}=a\left(t_{m}\right)$ is given by

$$
a_{m}=\left(\frac{6 \mathcal{C}}{|\Lambda|}\right)^{\frac{1}{3(1+w)}}
$$




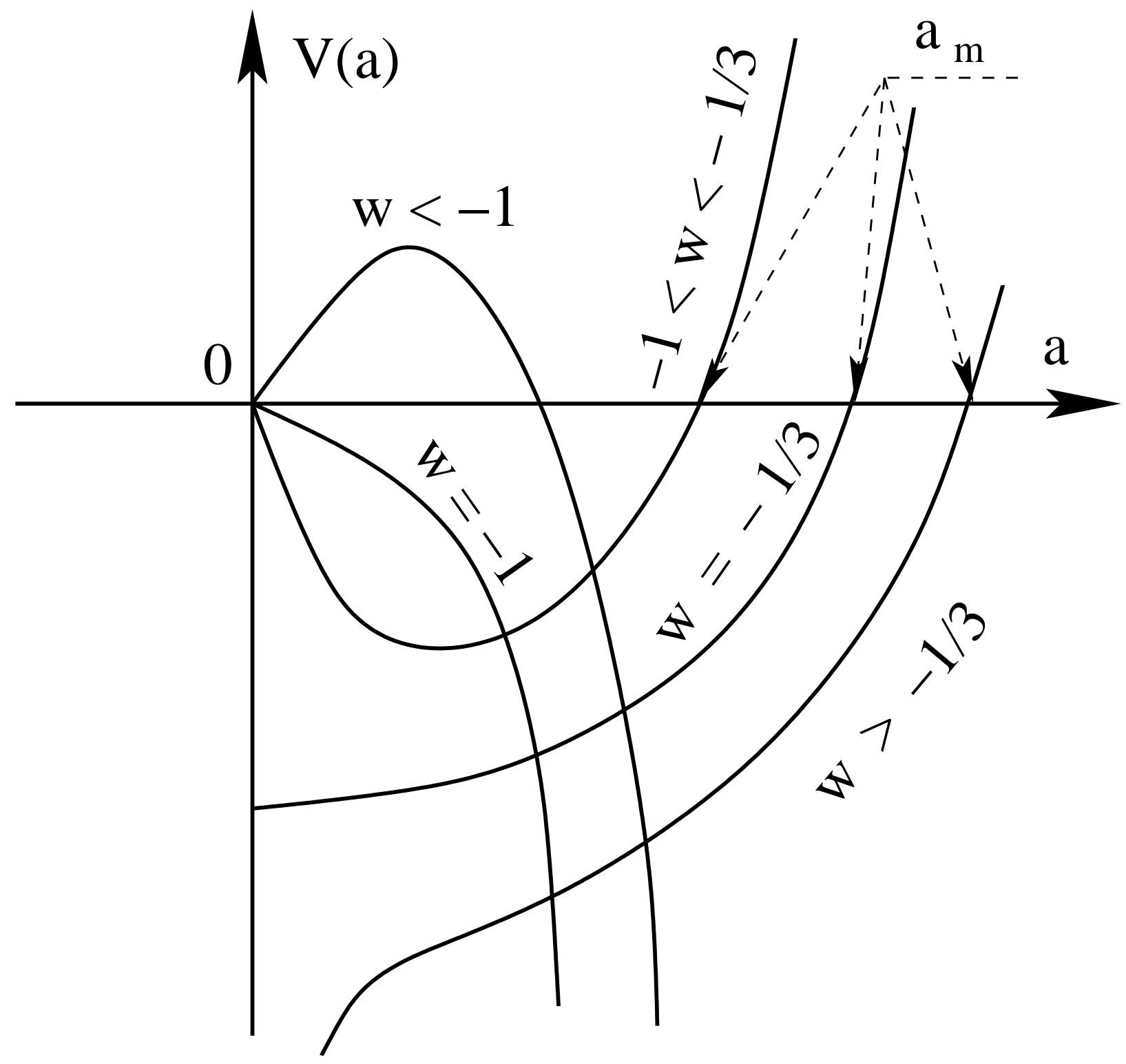

FIG. 5: The potential given by Eq.(2.27) for $k=0$ and $\Lambda<0$.

During the process, the universe is always decelerating,

$$
\ddot{a}=-\frac{d V(a)}{d a}<0
$$

as can be seen from Fig. 5 ,

Case A.3.2) $w=-\frac{1}{3}$ : In this case, we find

$$
\begin{aligned}
V(a) & =\frac{1}{6}|\Lambda| a^{2}-\mathcal{C}= \begin{cases}-\mathcal{C}, & a=0, \\
\infty, & a \rightarrow \infty,\end{cases} \\
\ddot{a} & =-\frac{d V(a)}{d a}=-\frac{1}{3}|\Lambda| a \leq 0 .
\end{aligned}
$$


Therefore, as in the last case, the universe expands from the big bang singularity at $a=0$ until its maximal radius $a_{m}$, given by Eq.(2.31) with $w=-1 / 3$ is reached. It then starts to collapse until a big crunch singularity is formed at $t=2 t_{m}$.

Case A.3.3) $-1<w<-\frac{1}{3}$ : In this case, from Fig. 5 we can see that $V(a=0)=$ $0=V\left(a_{m}\right)$, and the motion is also restricted to $a \leq a_{m}$. However, there is a fundamental difference between this case and the previous two cases: the potential $V(a)$ has a minimum at $a=a_{\text {min }}$, at which $d V\left(a_{\min }\right) / d a=0$. The universe accelerates initially. However, after expanding to $a=a_{m i n}$, it starts to decelerate until $a=a_{m}$, at which point the expansion velocity becomes zero. Afterward it will begin collapsing, until a big crunch singularity is developed at $a=0$, as shown in Fig. [6,

Case A.3.4) $w=-1$ : In this case, we have

$$
V(a)=-\frac{1}{6}|\Lambda| a^{2}\left(\frac{6 \mathcal{C}}{|\Lambda|}-1\right) .
$$

Therefore, there is a solution only when $\Lambda_{\text {eff }}>0$, for which the universe is de Sitter, and

$$
a(t)=e^{\sqrt{\Lambda_{e f f} / 3}\left(t-t_{0}\right)},
$$

where $\Lambda_{\text {eff }} \equiv 6 \mathcal{C}-|\Lambda|$.

Case A.3.5) $w<-1$ : In this case, there is a minimum $a_{\min }$ for which $V\left(a<a_{\min }\right) \geq 0$. Therefore, in contrast to the previous case, the motion of the universe is restricted to $a \geq$ $a_{m i n}$. As shown in Fig. 6, the universe starts to expand from $a=a_{m i n}$. Within a finite time $a\left(t_{s}\right)=\infty$, and a big rip singularity is formed.

B. $k=1$

In this case, the potential given by Eq.(2.11) can be written as

$$
V(a)=\frac{1}{2}-\frac{1}{6} \Lambda a^{2}\left[1+\left(\frac{\tilde{\mathcal{C}}}{a}\right)^{3(1+w)}\right],
$$

where $\tilde{\mathcal{C}} \equiv(6 \mathcal{C} / \Lambda)^{\frac{1}{3(1+w)}}$. As in the $k=0$ case, we distinguish the three cases, $\Lambda>0, \Lambda=0$ and $\Lambda<0$.

1. $\Lambda>0$

When $\Lambda>0$, it is convenient to further divide into the five sub-cases listed in Eq.(2.15). 


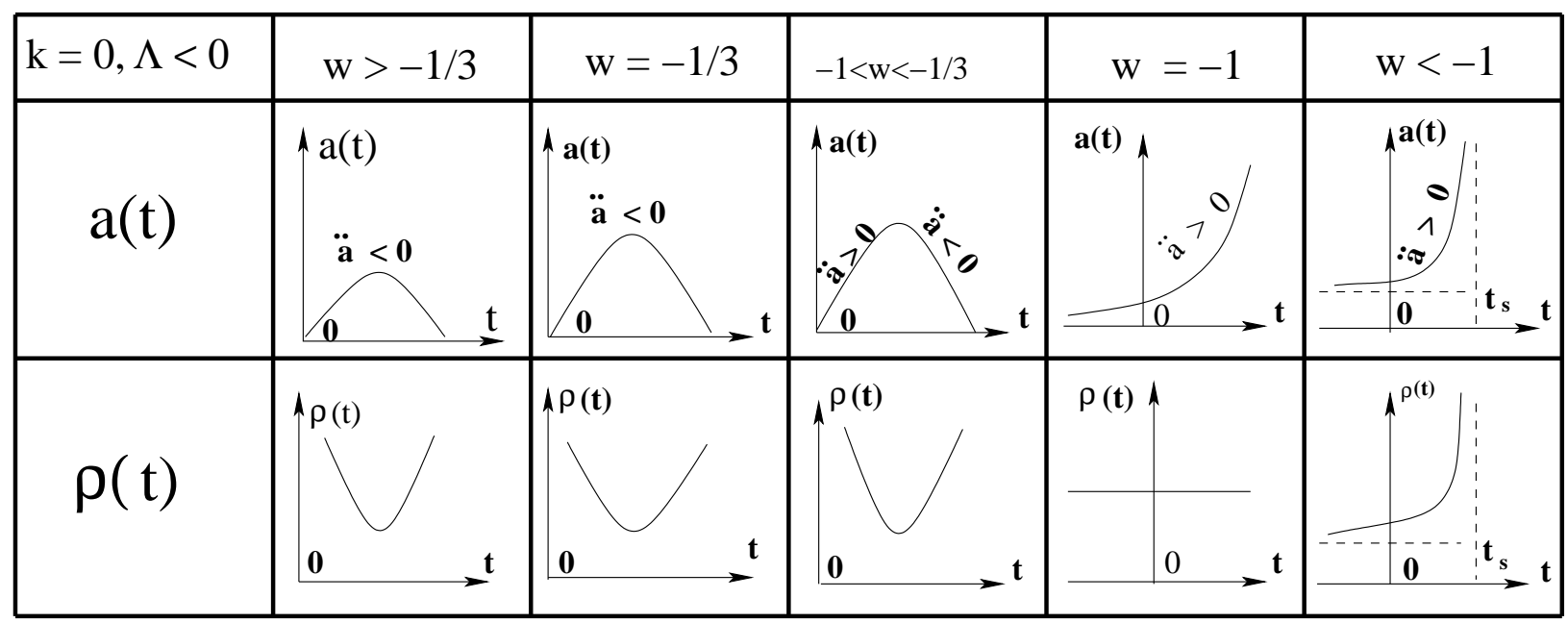

FIG. 6: The expansion factor $a(t)$, the acceleration $\ddot{a}(t)$, and the energy density $\rho(t)$ for $k=0$ and $\Lambda<0$. The spacetime is singular at $a=0$ for all cases with $w>-1$, (a big bang singularity). It is de Sitter for $w=-1$. When $w<-1$, a big rip singularity is developed at $t=t_{s}$, at which we have $a\left(t_{s}\right)=\rho\left(t_{s}\right)=\infty$.

Case B.1.1) $w>-\frac{1}{3}$ : In this case, it can be shown that for any given $w$ and $\rho_{0}$ there always exist a critical value $\Lambda_{c}$ and radius $a_{m}$ satisfying the conditions,

$$
V\left(a_{m}, w, \rho_{0}, \Lambda_{c}\right)=0, \quad V^{\prime}\left(a_{m}, w, \rho_{0}, \Lambda_{c}\right)=0
$$

where a prime denotes ordinary differentiation with respect to $a$. The solutions for these conditions are

$$
\begin{aligned}
a_{m} & =[3(1+w) \mathcal{C}]^{\frac{1}{1+3 w}} \\
\Lambda_{c} & =\left(\frac{1+3 w}{1+w}\right)[3(1+w) \mathcal{C}]^{-\frac{2}{1+3 w}}
\end{aligned}
$$

As shown below, the solutions with $\Lambda>\Lambda_{c}$ have quite different properties from the ones with $\Lambda<\Lambda_{c}$. Therefore, we further distinguish the three cases, $\Lambda>\Lambda_{c}, \Lambda=\Lambda_{c}$ and $\Lambda<\Lambda_{c}$.

Case B.1.1.1) $w>-\frac{1}{3}, \Lambda>\Lambda_{c}$ : In this case, the potential $V(a)$ is always negative for any given $a$, as shown in Fig. 17. Therefore, the corresponding solutions have no turning points. If the universe initially starts to expand from a big bang singularity at $a=0$, it will expand forever, as shown by Fig. 8. However, the potential has a maximum at $a=a_{m}$, for which we have

$$
\ddot{a}= \begin{cases}<0, & a<a_{m}, \\ =0, & a=a_{m}, \\ >0, & a>a_{m},\end{cases}
$$




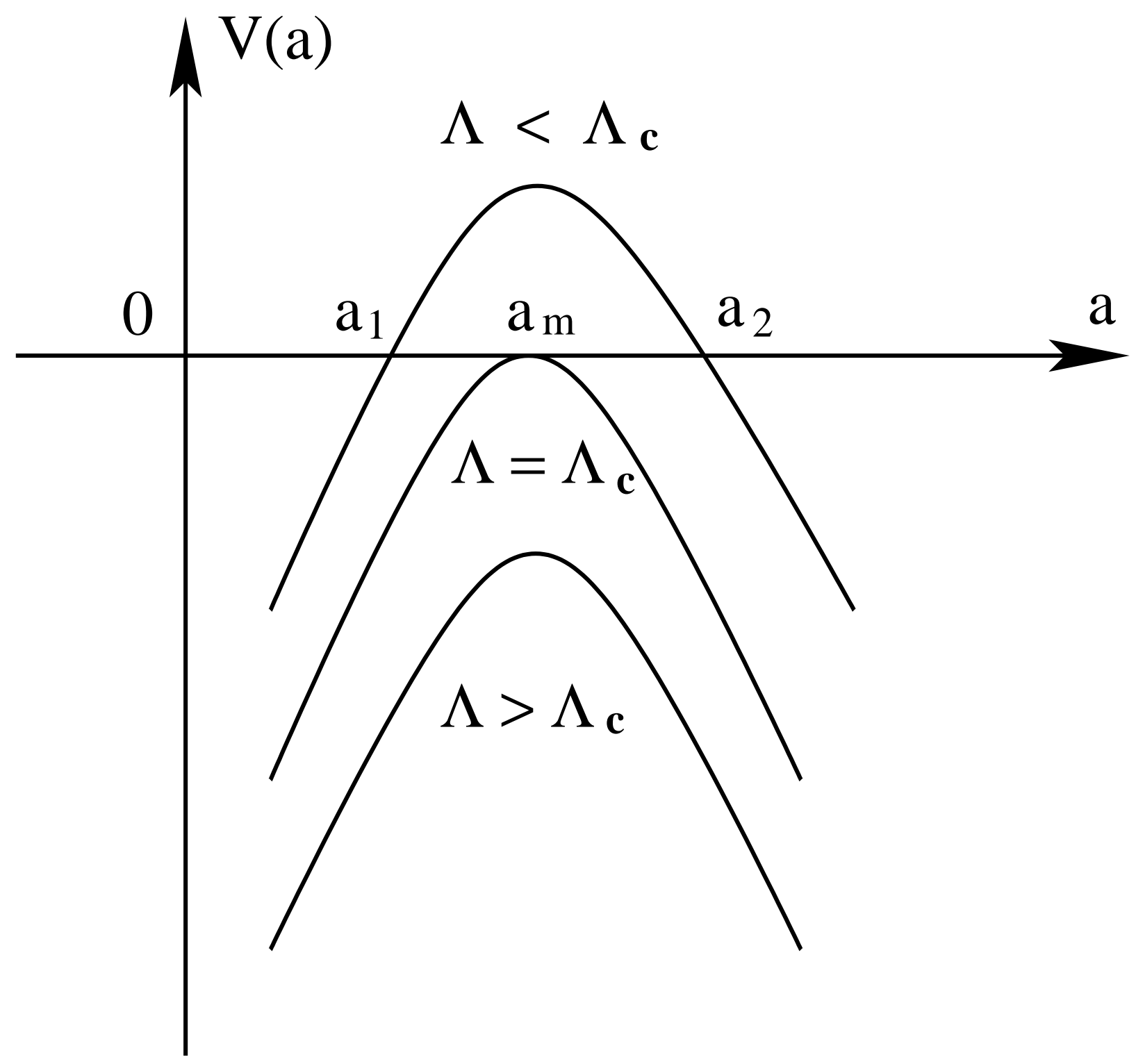

FIG. 7: The potential given by Eq.(2.36) for $k=1, w>-\frac{1}{3}$ and $\Lambda>0$, where $\Lambda_{c}=\Lambda_{c}\left(w, \rho_{0}\right)$.

that is, the universe is initially decelerating. Once it expands to $a=a_{m}$, the expansion begins to accelerate.

Case B.1.1.2) $w>-\frac{1}{3}, \Lambda=\Lambda_{c}$ : In this case, there exists a static point $a_{m}$, at which we have $V\left(a_{m}\right)=V^{\prime}\left(a_{m}\right)=0$, as seen in Fig. 7. Therefore, if the universe starts to expand from the big bang at $a=0$, it will expand until $a=a_{m}$. The universe is decelerating during this period. At the point $a=a_{m}$, the universe becomes static, since $\dot{a}=0=\ddot{a}$. However, this point is not stable, and with small perturbations, the universe will either collapse until a singularity is developed at $a=0$ or expand forever with $\ddot{a}>0$. If the universe initially has 


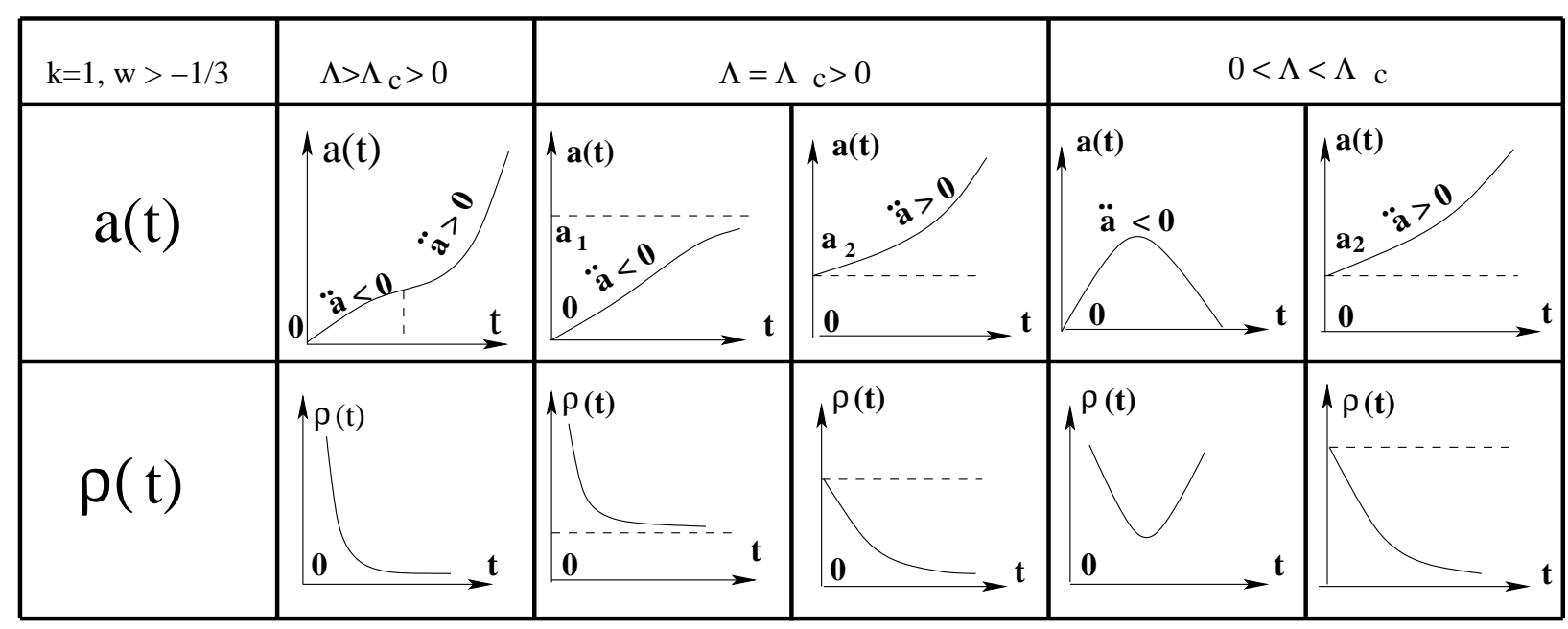

FIG. 8: The expansion factor $a(t)$, the acceleration $\ddot{a}(t)$, and the energy density $\rho(t)$ for $k=1, w>$ $-\frac{1}{3}$ and $\Lambda>0$, where $\Lambda_{c}$ is given by Eq.(2.38). A big bang singularity occurs at $a=0$ in all cases with $\Lambda \geq \Lambda_{c}$. In the first sub-case of $0<\Lambda<\Lambda_{c}$, both big bang and big crunch singularities occur, while in the second sub-case, spacetime is free of any kind of spacetime singularities.

an acceleration $a=a_{i}>a_{m}$, then as shown in Fig. 7, it will expand forever. Since $V^{\prime}(a)$ is always negative, the universe in this region always accelerates.

Case B.1.1.3) $w>-\frac{1}{3}, 0<\Lambda<\Lambda_{c}$ : In this case, $V(a)=0$ has two real and positive roots, $a_{1}$ and $a_{2}$, as shown in Fig. 7. Without loss of generality, we assume that $a_{2}>a_{1}$. Since $V(a)>0$ for $a \in\left(a_{1}, a_{2}\right)$, the motion is forbidden in this region. As in the previous case, depending on the initial conditions, the universe can evolve very differently. In particular, if it starts to expand from the big bang singularity at $a=0$, it will expand until $a=a_{1}$, where $\dot{a}=0$ and $\ddot{a}<0$. Since $\ddot{a}<0$ at this point, the universe will start collapsing, until a big crunch singularity is developed at $a=0$. If the universe starts to expand at $a_{i} \geq a_{2}$, it will expand forever. In the latter case, the universe is always accelerating, as can be seen in Fig. 7.

Case B.1.2) $w=-\frac{1}{3}$ : In this case, we have

$$
V(a)=\frac{1}{2}(1-2 \mathcal{C})-\frac{1}{6} \Lambda a^{2} .
$$

Thus, the motion of the universe will differ depending on the value of $\mathcal{C}\left(\rho_{0}\right)$. In particular, when $\mathcal{C}\left(\rho_{0}\right)<1 / 2$, there exists a minimal $a_{\text {min }}$, for which $V\left(a<a_{\text {min }}\right)>0$; that is, the motion in the region $0<a<a_{\text {min }}$ is forbidden, as shown in Fig. 9. When $\mathcal{C}\left(\rho_{0}\right) \geq 1 / 2$, the universe starts to expand from the big bang singularity at $a=0$. In all cases we have 


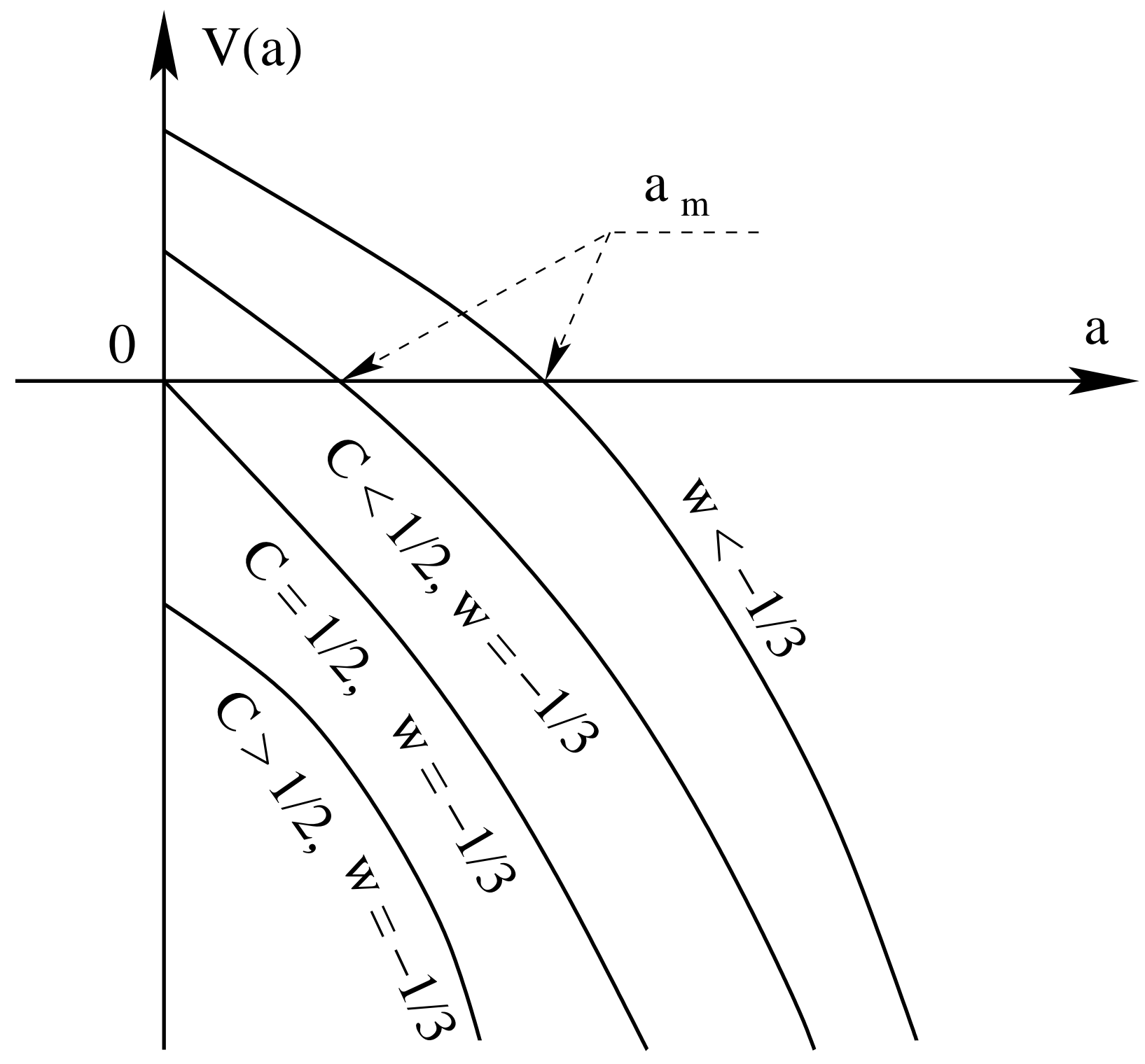

FIG. 9: The potential given by Eq.(2.40) for $k=1, w \leq-\frac{1}{3}$ and $\Lambda>0$.

$V^{\prime}(a)<0$, so that the universe is always accelerating [cf. Fig. 10].

Case B.1.3) $-1<w<-\frac{1}{3}$ : In this case, we find that $V^{\prime}(a)$ is strictly negative for any $a \geq 0$ with $V(0)=1 / 2$. Therefore, similar to the case $w=-1 / 3$ and $\mathcal{C}<1 / 2$, there exists a minimal $a_{\text {min }}$, for which $V\left(a<a_{\text {min }}\right)>0$, and the motion in the region $0<a<a_{\text {min }}$ is forbidden, as shown in Fig. 9. Thus, in the present case, the universe starts to expand from a radius $a_{i} \geq a_{\min }$ until $a=\infty$ without a turning point. Since $V^{\prime}(a)<0$ for any $a \geq a_{\min }$, the universe is always accelerating. No singularities develop: not big bang, big crunch, nor big rip, as shown in Fig. 10. 


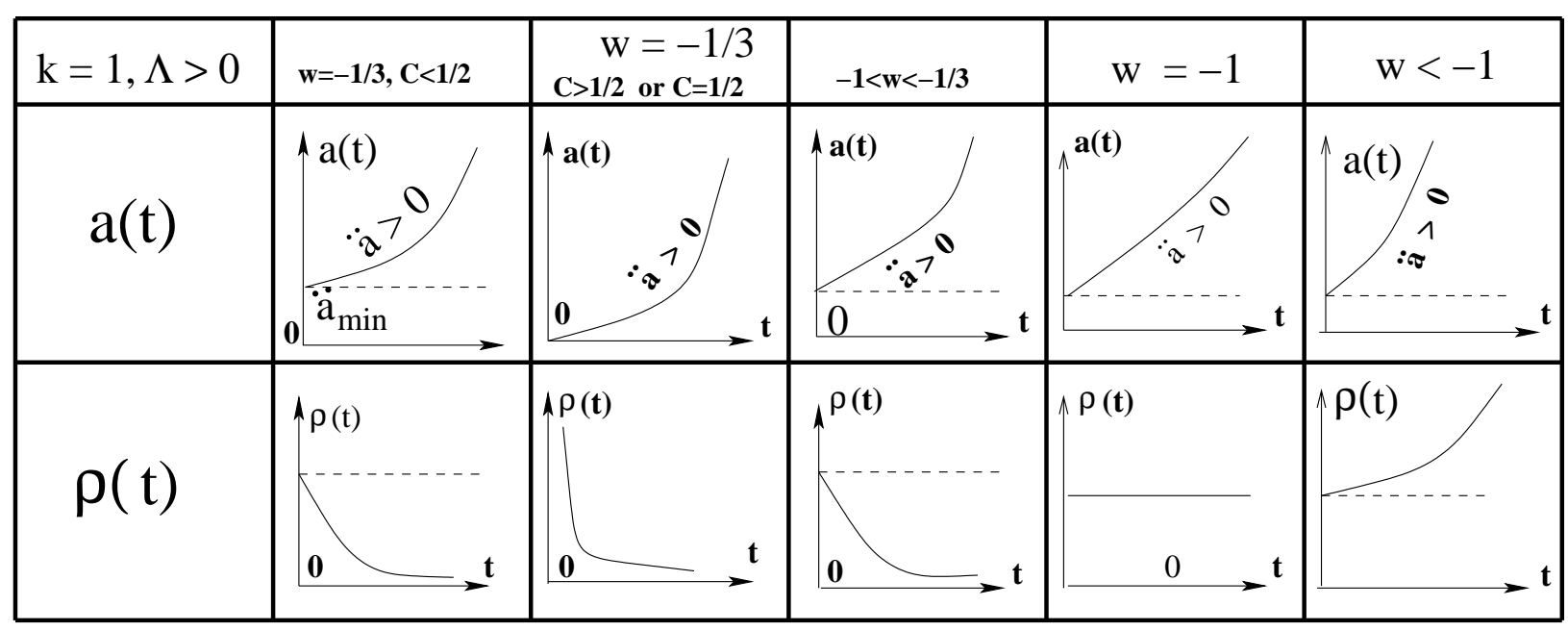

FIG. 10: The expansion factor $a(t)$, the acceleration $\ddot{a}(t)$, and the energy density $\rho(t)$ for $k=$ $1, w \leq-\frac{1}{3}$ and $\Lambda>0$. A big bang singularity occurs only in the case $w=-1 / 3$ and $\mathcal{C} \geq 1 / 2$. In the case $w<-1$, a big rip singularity occurs at $a=\infty$.

Case B.1.4) $w=-1$ : In this case, the potential is simply parabolic,

$$
V(a)=\frac{1}{2}-\frac{1}{6}(\Lambda+6 \mathcal{C}) a^{2}
$$

schematically shown by the top curve in Fig. 9. As a result, the motion is similar to the last case, except that now $\rho=\rho_{0}$.

Case B.1.5) $w<-1$ : In this case, we also have $V^{\prime}(a)<0$ and there exists a finite radius, $a_{\text {min }}$, such that when $a<a_{\text {min }}, V(a)>0$, and when $a \geq a_{\text {min }}, V(a) \leq 0$. The only difference is that in the present case, a big rip singularity develops at $a=\infty$, since $\rho \propto a^{3(|w|-1)}$, as shown in Fig. 10.

$$
\text { 2. } \Lambda=0
$$

In this case, we find that

$$
V(a)=\frac{1}{2}-\frac{\mathcal{C}}{a^{1+3 w}}
$$

Fig. 11 shows the potential for various values of $w$. For $w>-1 / 3$, the motion of the universe is restricted to $a \leq a_{m}$, where $a_{m}$ is the solution of $V(a)=0$. The universe starts to expand at the big bang singularity $a=0$ until the turning point $a=a_{m}$ is reached. It then starts to collapse, until a big crunch singularity is developed at $a=0$, as shown in Fig. 12 . 


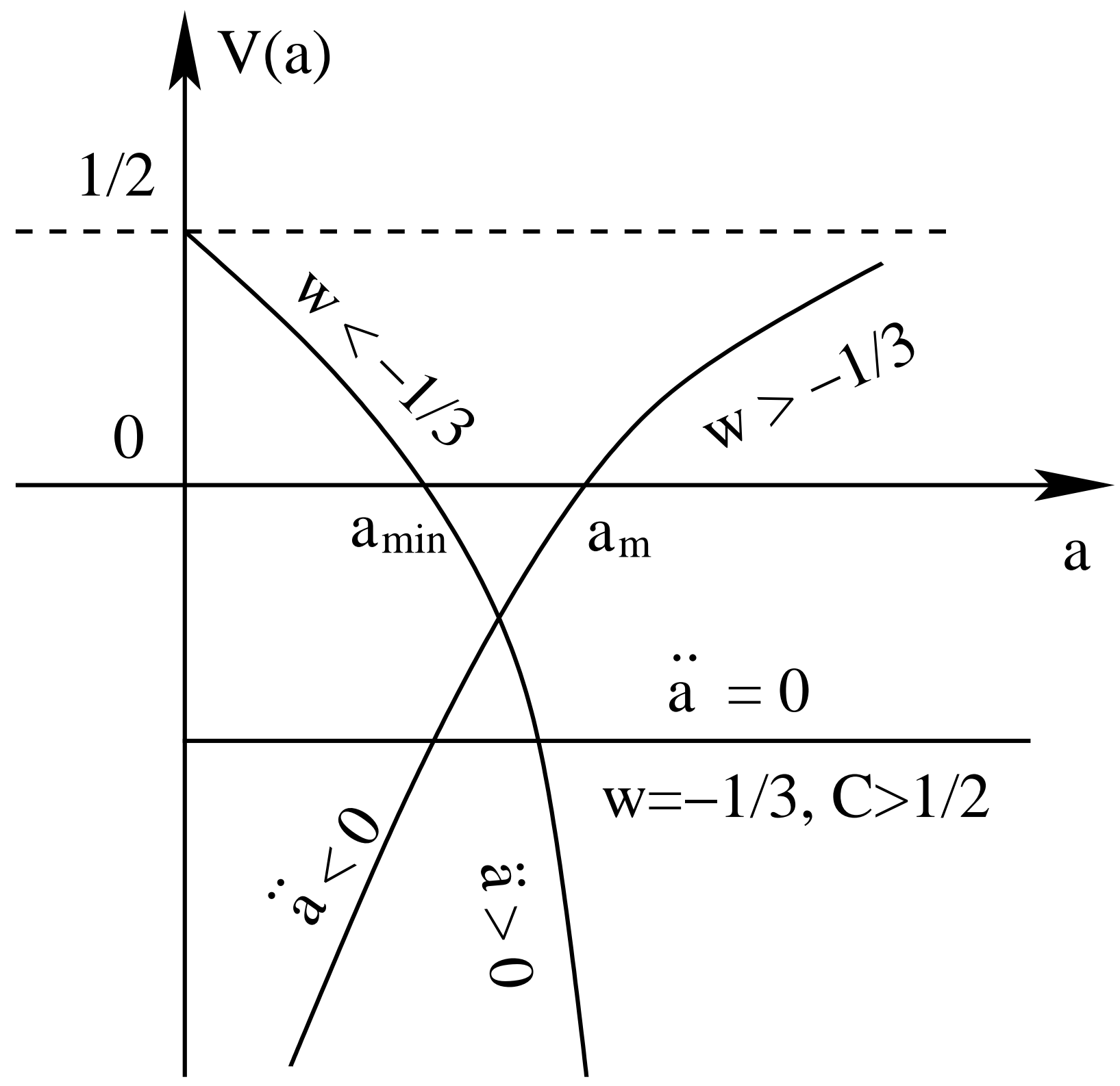

FIG. 11: The potential given by Eq.(2.42) for $k=1$ and $\Lambda=0$.

When $w=-1 / 3$, there is motion only for $\mathcal{C}>1 / 2$, for which the universe expands linearly from a big bang singularity at $a=0$ with $\ddot{a}=0$.

When $-1 \leq w<-1 / 3$, motion is possible only for $a>a_{\min }$, as shown in Figs. 11 and 12. The universe starts to expand from the initial point $a_{i} \geq a_{\min }$ with $\ddot{a}>0$. No turning point exists; so, the universe will expand forever. In this case, the matter density remains finite during the whole process, so no singularity exists.

When $w<-1$, it can be shown that the motion for $a<a_{\min }$ is also forbidden. As a 


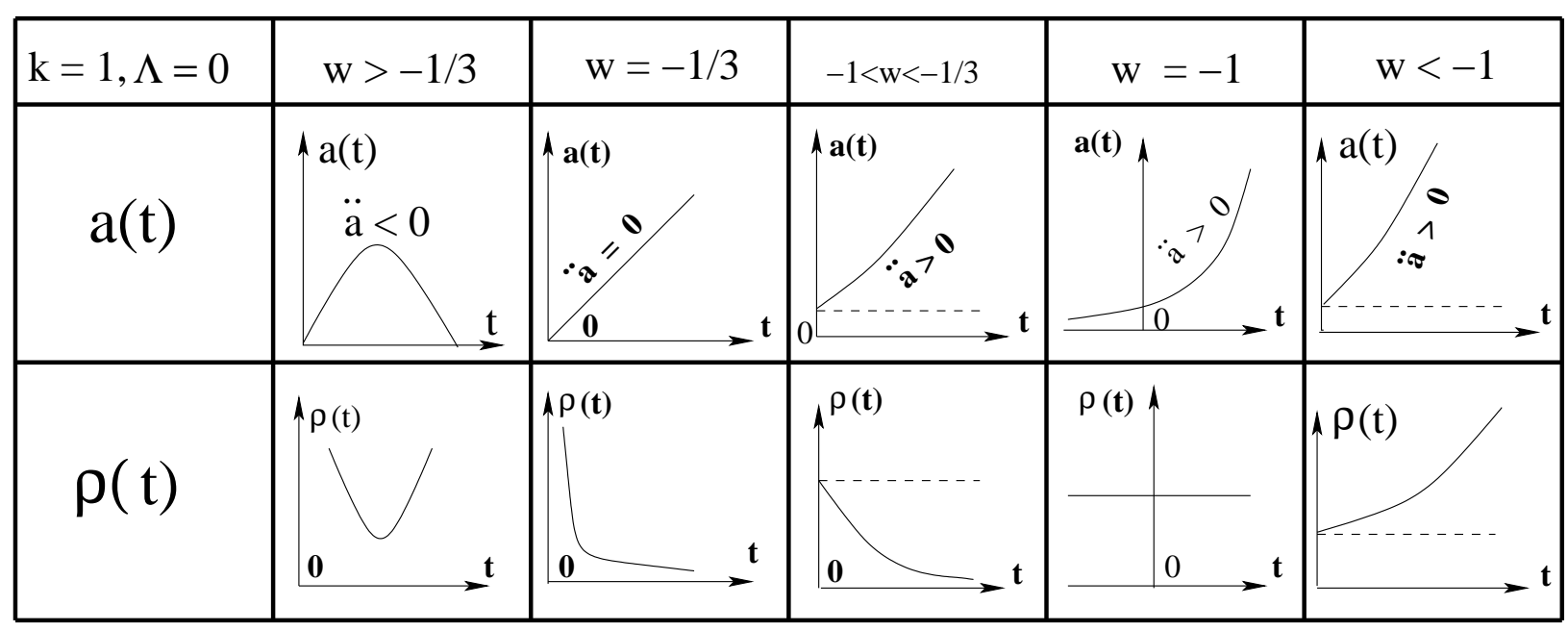

FIG. 12: The expansion factor $a(t)$, the acceleration $\ddot{a}(t)$, and the energy density $\rho(t)$ for $k=1$ and $\Lambda=0$. There are both big bang and big crunch singularities in the case $w>-1 / 3$, while only a big bang singularity occurs in the case $w=-1 / 3$. There is no singularity in the cases with $-1 \leq w<-1 / 3$. A big rip singularity occurs at $a=\infty$ for $w<-1$.

result, no big bang singularity exists; however, a big rip singularity develops as $a \rightarrow \infty$, as shown by Fig. 12, During the whole process, $\ddot{a}>0$.

3. $\Lambda<0$

In this case, we have

$$
V(a)=\frac{1}{2}+\frac{1}{6}|\Lambda| a^{2}-\frac{\mathcal{C}}{a^{1+3 w}}
$$

which has the properties as shown in Fig. 13. In particular, when $w>-1 / 3$, the universe starts to expand from a big bang singularity at $a=0$ until a maximal radius $a_{m}$ is reached, where $V\left(a_{m}\right)=0$. Afterward, the universe starts collapsing, reaching a big crunch singularity at $t=2 t_{m}$, where $t_{m}$ is determined by $a_{m}=a\left(t_{m}\right)$. Throughout the process the universe is decelerating, as shown in Fig. 14.

When $w=-1 / 3$, the potential is non-negative for $\mathcal{C} \leq 1 / 2$, so the motion is forbidden. When $\mathcal{C}>1 / 2$ we have $V(a)<0$ for $a<a_{m}$, where $a_{m}$ is the root of $V(a)=0$, as shown in Fig. 13. Thus, motion is possible in the region $a<a_{m}$, for which the universe expands from a big bang singularity at $a=0$. Once the maximal radius $a_{m}$ is reached, the universe collapses until a big crunch is developed at $t=2 t_{m}$. 
When $-1<w<-1 / 3$, the potential is negative only for $a<a_{m}$, as shown in Fig. 13. In particular, a big bang (crunch) singularity occurs at $t=0\left(t=2 t_{m}\right)$. The difference from the previous case is that there now exists a time $t_{\text {max }}$, such that the universe is accelerating for $0<t<t_{\max }$ and $2 t_{m}-t_{\max }<t<2 t_{m}$, while during the time $t_{m}-t_{\max }<t<2 t_{m}-t_{\max }$ the universe is decelerating. $t_{\max }$ is the root of $V^{\prime}\left(t_{\max }\right)=0$.

When $w=-1$, the potential is non-positive only for $\mathcal{C}>|\Lambda| / 6$ and $a \geq a_{\text {min }}$, where $a_{\text {min }}$ is the root of $V(a)=0$, as shown in Fig. 13. In this case the universe starts to expand at an initial radius $a_{i} \geq a_{m i n}$, and will expand forever with $\ddot{a}>0$. However, spacetime is not singular even when $a=\infty$.

When $w<-1$, the potential is non-positive only for $a \geq a_{\min }$, where $a_{\min }$ is again the root of $V(a)=0$, as shown in Fig. 13. The evolution of the universe in this case is similar to the last one, except that now a big rip singularity develops at $a=\infty$.

C. $k=-1$

When $k=-1$, the potential is given by

$$
V(a)=-\frac{1}{2}-\frac{1}{6} \Lambda a^{2}-\frac{\mathcal{C}}{a^{1+3 w}}
$$

To study the motion of the universe in this case, it is also convenient to distinguish the three cases $\Lambda>0, \Lambda=0$ and $\Lambda<0$. In each case there are five sub-cases for different choices of $w$.

\section{1. $\Lambda>0$}

In this case, we find that $V(a) \rightarrow-\infty$ as $a \rightarrow \infty$, and

$$
V(a \rightarrow \infty)= \begin{cases}-1 / 2, & w<-1 / 3 \\ -(1 / 2+\mathcal{C}), & w=-1 / 3 \\ -\infty, & w>-1 / 3\end{cases}
$$

when $a \rightarrow 0$, as shown by Fig. 15. Thus, when $w>-1 / 3$, the potential has a maximum at $a_{m}$ where $V^{\prime}\left(a_{m}\right)=0$. The universe expands from a big bang at $a=0$. Initially, it is decelerating since $\ddot{a}<0$. However, after expanding to $a_{m}$, the expansion begins accelerating, since now $\ddot{a}>0$, as shown in Fig. 16. When $-1<w \leq-1 / 3$, the universe expands from 


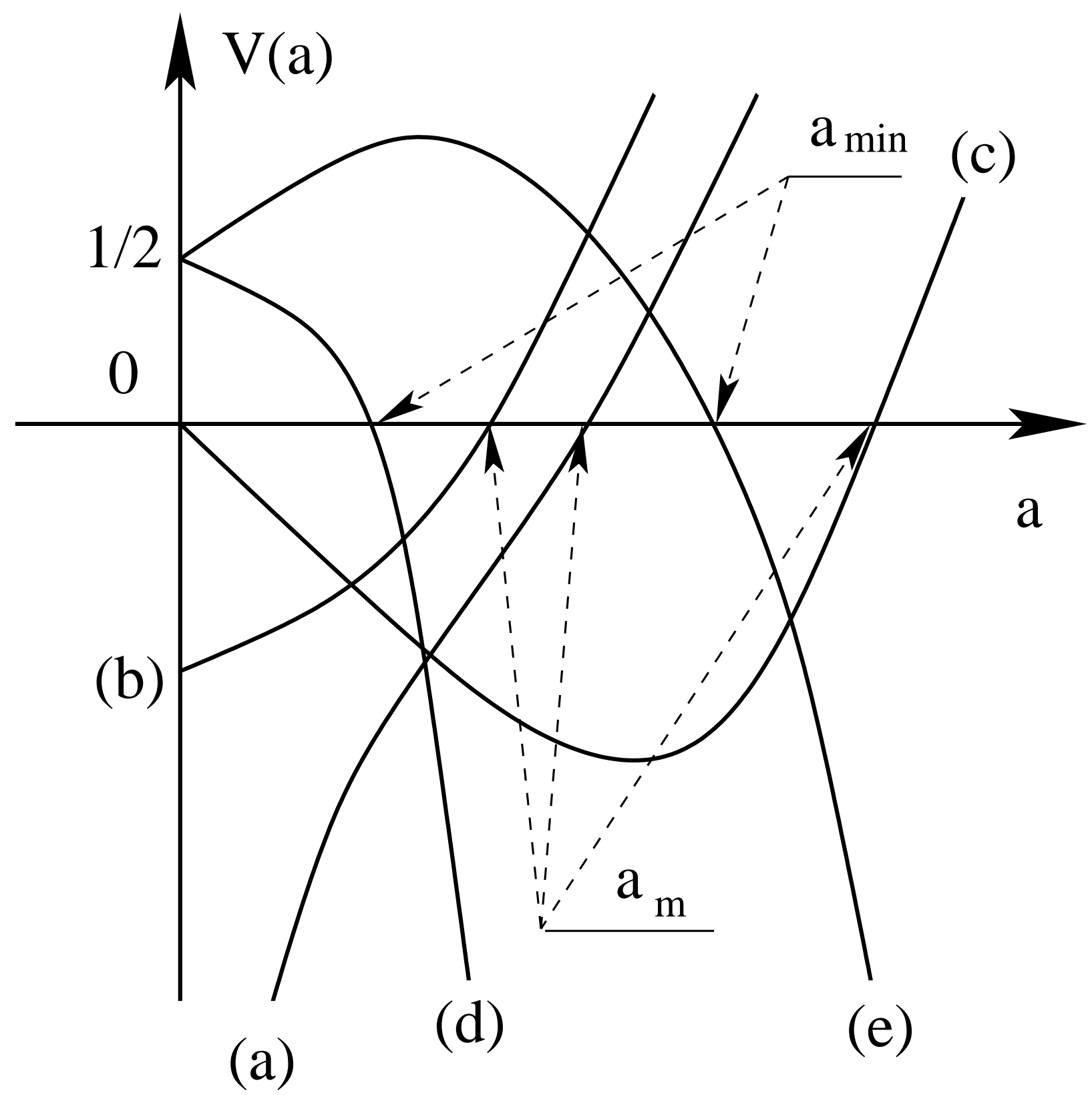

FIG. 13: The potential given by Eq.(2.43) for $k=1$ and $\Lambda=0$ : (a) for $w>-1 / 3$; (b) for $w=-1 / 3$ and $\mathcal{C}>1 / 2 ;(\mathrm{c})$ for $-1<w<-1 / 3$; (d) for $w=-1$ and $\mathcal{C}>|\Lambda| / 6$; and (e) for $w<-1$.

a big bang at $a=0$ until $a=\infty$, without a turning point since $\ddot{a}>0$ during the whole process. The case of $w=-1$ is similar to the case of $-1<w \leq-1 / 3$, except that spacetime is not singular either at $a=0$ or at $a=\infty$, as shown in Fig.16. When $w<-1$, the universe starts to expand from $a=0$ with $\ddot{a}>0$ for any given $a$. There is no big bang singular at $a=0$; however, there is a big rip singularity at $a=\infty$. 


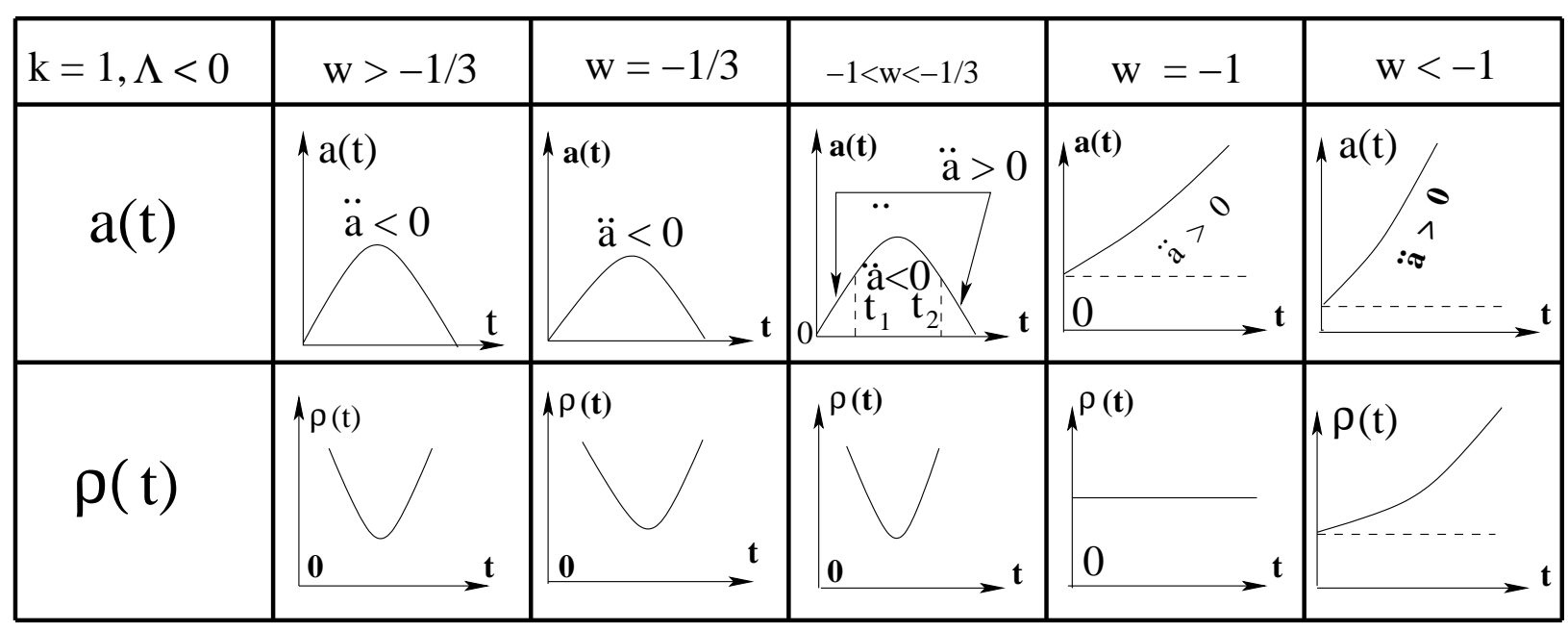

FIG. 14: The expansion factor $a(t)$, the acceleration $\ddot{a}(t)$, and the energy density $\rho(t)$ for $k=1$ and $\Lambda<0$. There are both big bang and big crunch singularities for the case $w>-1$, while only a big bang singularity occurs for the case $w=-1$. There are no singularities for the case $w=-1$, while there is a big rip singularity at $a=\infty$ for $w<-1$.

2. $\Lambda=0$

In this case, we have

$$
\begin{aligned}
& V(a)=-\frac{1}{2}-\frac{\mathcal{C}}{a^{1+3 w}}<0, \\
& \ddot{a}=-\frac{(3 w+1) \mathcal{C}}{a^{2+3 w}}= \begin{cases}<0, & w>-1 / 3, \\
0, & w=-1 / 3, \\
>0, & w<-1 / 3,\end{cases}
\end{aligned}
$$

when $a \in[0, \infty)$, as shown by Fig. 17. We also have

$$
\rho(a)= \begin{cases}\infty, & w>-1 / 3, \\ \rho_{0}, & w=-1 / 3, \\ 0, & w<-1 / 3,\end{cases}
$$

as $a \rightarrow 0$, and

$$
\rho(a)= \begin{cases}0, & w>-1 / 3, \\ \rho_{0}, & w=-1 / 3, \\ \infty, & w<-1 / 3,\end{cases}
$$

as $a \rightarrow \infty$. Fig. 18 shows the motion of the universe for each given $w$. 


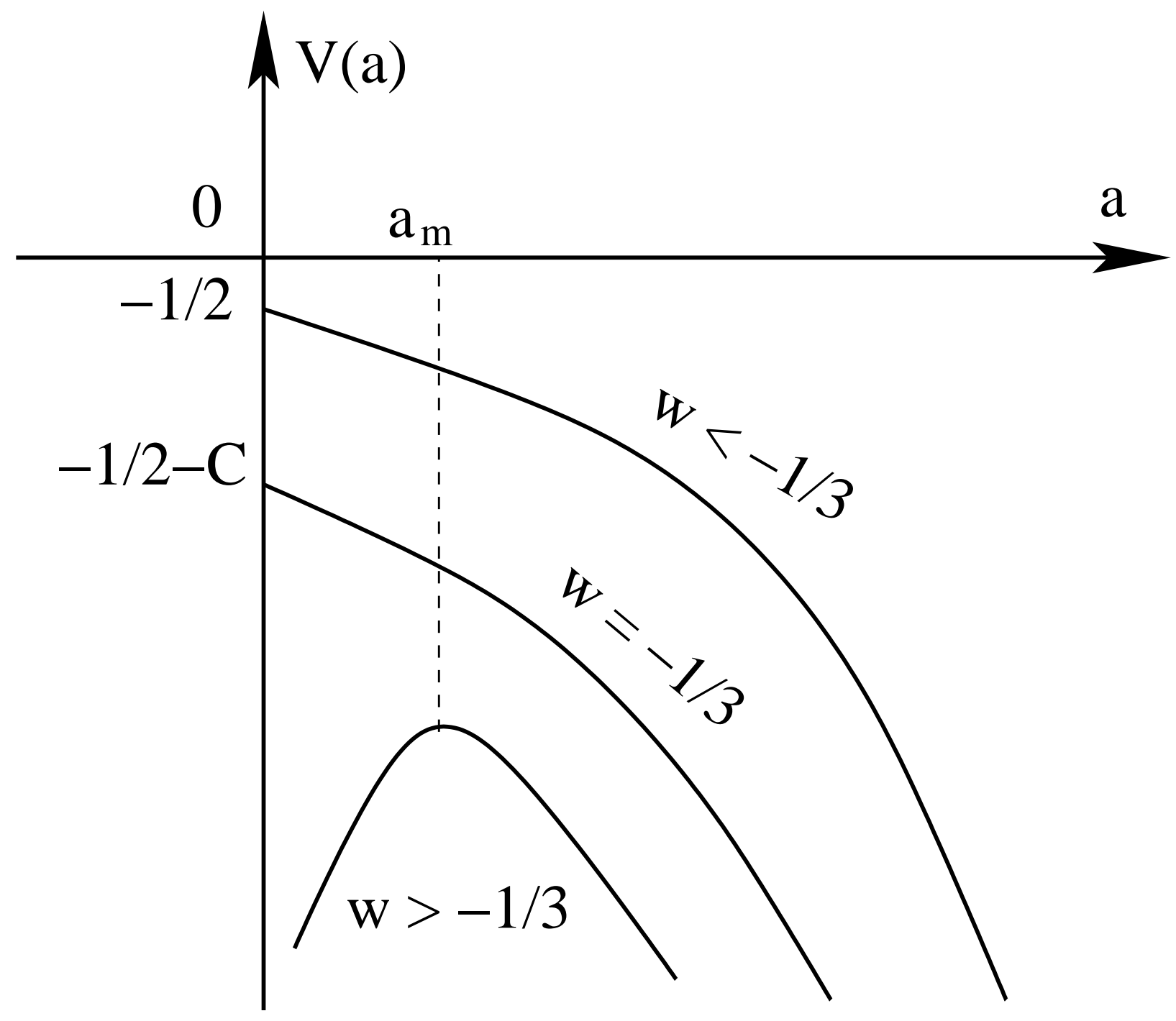

FIG. 15: The potential given by Eq.(2.44) for $k=-1$ and $\Lambda>0$.

3. $\Lambda<0$

In this case, we have

$$
V(a)=-\frac{1}{2}+\frac{1}{6}|\Lambda| a^{2}-\frac{\mathcal{C}}{a^{1+3 w}} .
$$

Depending on the values of $\Lambda, \mathcal{C}$ and $w$, the potential will have quite different properties. In the following we sstudy them case by case.

Case C.3.1) $w>-1 / 3$ : In this case, the potential, shown schematically in Fig. 19, is non-positive only for $a \leq a_{m}$, where $a_{m}$ is the positive root of $V(a)=0$. Clearly, there is a big bang singularity at $a=0$, from which the universe expands until $a=a_{m}$. Afterward, it collapses to a big crunch singularity at $t=2 t_{m}$, for which $a\left(2 t_{m}\right)=0$, as shown in Fig. 20. 


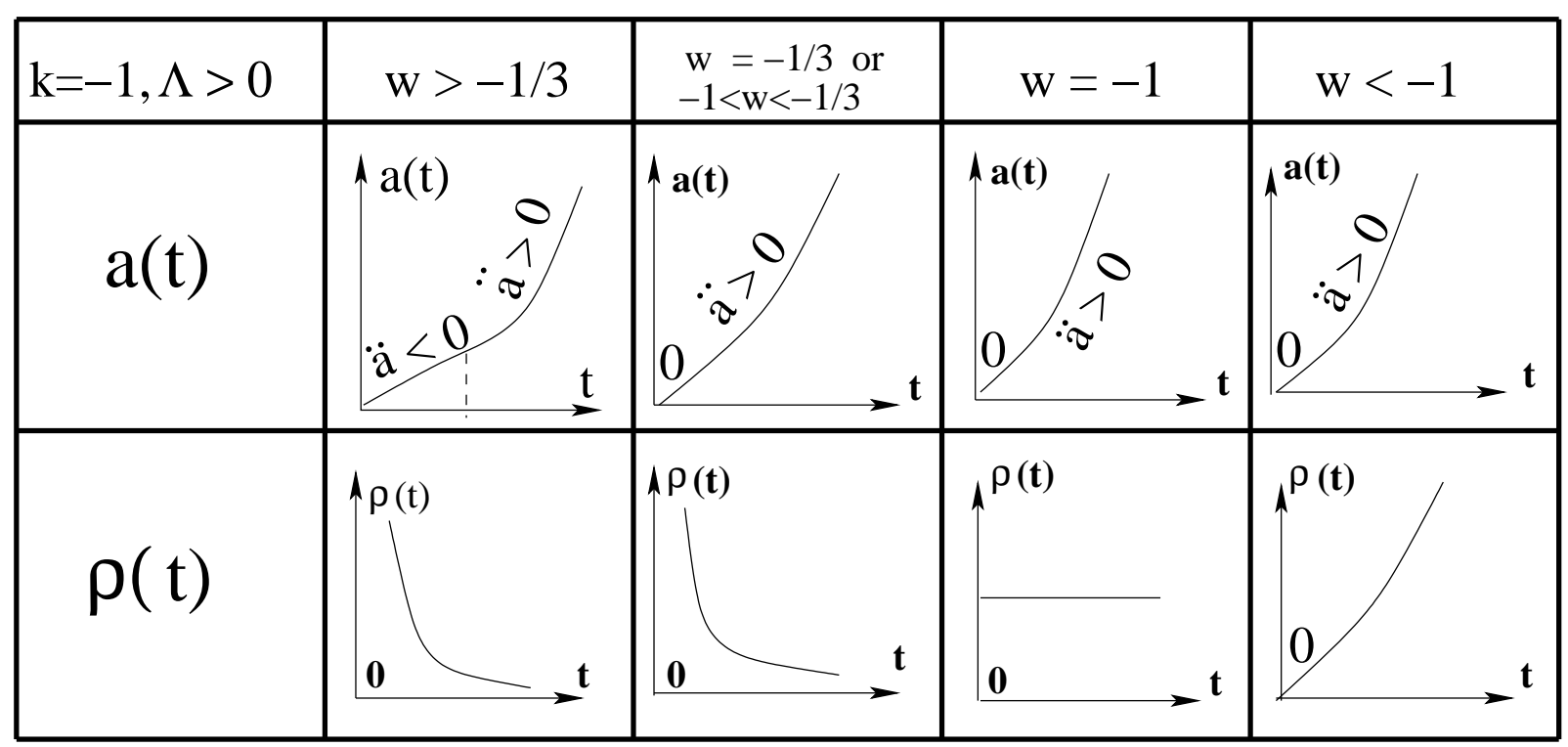

FIG. 16: The expansion factor $a(t)$, the acceleration $\ddot{a}(t)$, and the energy density $\rho(t)$ for $k=-1$ and $\Lambda>0$. There is a big bang singularity for $w>-1$, no singularity for $w=-1$, and a big rip singularity at $a=\infty$ for $w<-1$.

Case C.3.2) $w=-1 / 3$ : The potential is similar to the previous case, except that now $V(0)=-1 / 2-\mathcal{C}$, as shown in Fig. 19. The resulting motion of the universe is qualitatively similar to the previous case, as shown in Fig. 20.

Case C.3.3) $-1<w<-1 / 3$ : In this case the potential has a minimum at $a=a_{m i n}$, as shown in Fig. 19, for which $\ddot{a}<0$ for $a<a_{\text {min }}$, and $\ddot{a}>0$ for $a>a_{\text {min }}$, as shown in Fig. 20.

Case C.3.4) $w=-1$ : In this case, depending on the ratio $6 \mathcal{C} /|\Lambda|$, three sub-cases may be distinguished. When $6 \mathcal{C} /|\Lambda|<1$, the potential is non-positive only when $a \leq a_{m}$, where $a_{m} \equiv[3 /(|\Lambda|-6 \mathcal{C})]^{1 / 2}$, as shown in Fig. 21. The universe expands from $a=0$ until $a=a_{m}$, and then collapses until $a=0$ again. No spacetime singularity is developed; thus, the universe oscillate between $a=0$ and $a=a_{m}$, as shown in Fig. 22,

When $6 \mathcal{C} /|\Lambda|=1$, we find that $V(a)=-1 / 2$, and the universe expands linearly starting from $a=0$. There is no turning point or spacetime singularity, as shown in Figs. 21 and 22 ,

When $6 \mathcal{C} /|\Lambda|>1$, we find that $V(a)<-1 / 2$ for any given $a$. Starting from $a=0$, the universe expands always at an ever accelerating rate $(\ddot{a}>0)$ until $a=\infty$, as shown in Figs. 21 and 22. No spacetime singularity is developed during the whole process.

Case C.3.5) $w<-1$ : In this case, it can be shown that for any given $\rho_{0}$ there exists a 


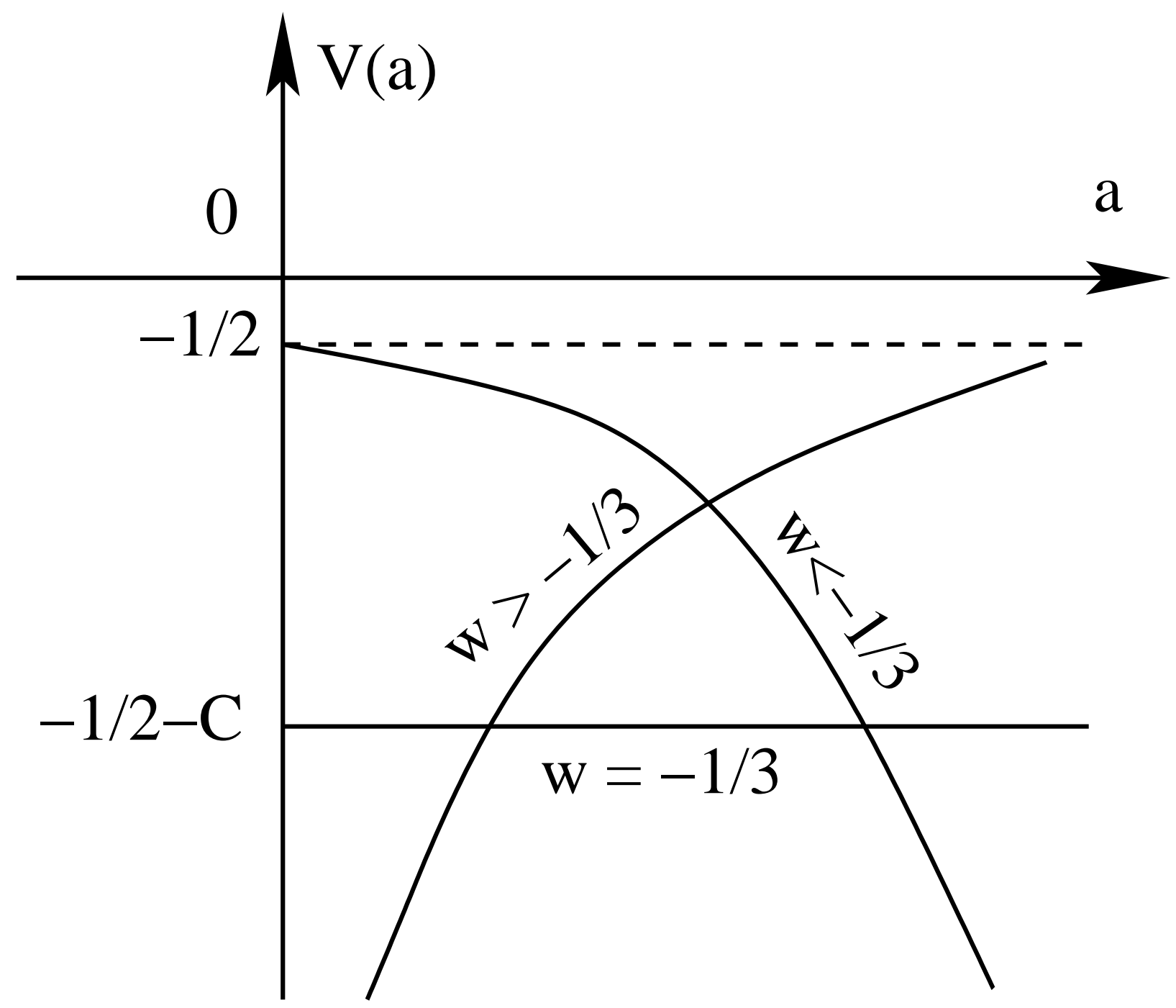

FIG. 17: The potential given by Eq.(2.46) for $k=-1$ and $\Lambda=0$.

critical value $\Lambda_{c}$ such that $V(a)=0$ has two root positive roots for $|\Lambda|>\left|\Lambda_{c}\right|$, one positive root for $|\Lambda|=\left|\Lambda_{c}\right|$, and no positive roots for $|\Lambda|<\left|\Lambda_{c}\right|$, as shown in Fig,23, where $\Lambda_{c}$ is the solution of the equations $V\left(a_{m}, \Lambda_{c}\right)=0$, and $V^{\prime}\left(a_{m}, \Lambda_{c}\right)=0$. It can be shown that $\Lambda_{c}$ is given by,

$$
\Lambda_{c}=\left(\frac{3|w|-1}{|w|-1}\right)[3(|w|-1) \mathcal{C}]^{\frac{2}{3|w|-1}} .
$$

Case C.3.5.1) $|\Lambda|>\left|\Lambda_{c}\right|$ : In this case, the potential is positive in the region $a_{1}<a<a_{2}$, where $a_{1}$ and $a_{2}$ are the two positive roots of $V(a)=0$ with $a_{2}>a_{1}$. Therefore, the motion of the universe is now restricted to the regions $0 \leq a \leq a_{1}$ and $a \geq a_{2}$, depending on the initial conditions. If the universe starts to expand at $a=0$, it will expand until reaching its maximal radius $a=a_{1}$, and then collapse until $a=0$. During the whole process, $\ddot{a}<0$. 


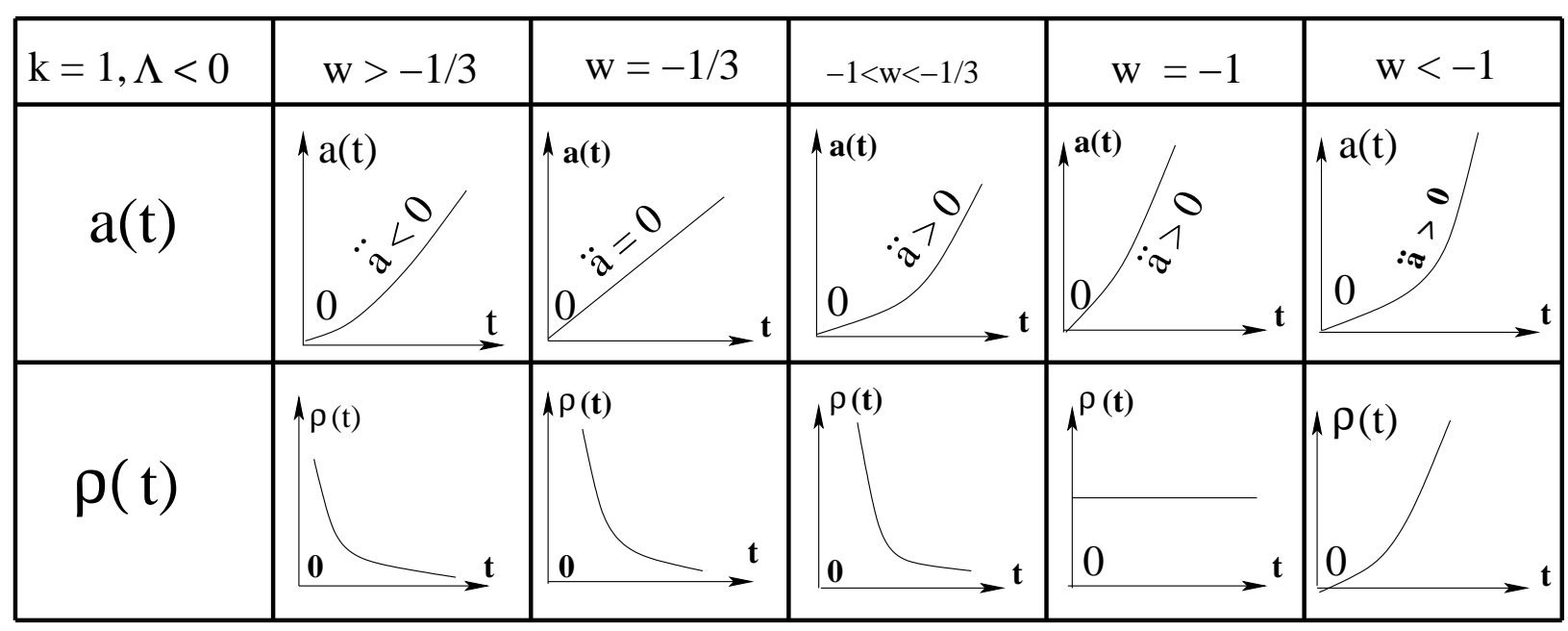

FIG. 18: The expansion factor $a(t)$, the acceleration $\ddot{a}(t)$, and the energy density $\rho(t)$ for $k=-1$ and $\Lambda=0$. There is a big bang singularity for $w>-1$, no singularity for $w=-1$, and a big rip singularity at $a=\infty$ for $w<-1$.

Since spacetime is not singular at $a=0$, the universe again starts to expand. This process will repeat endlessly, as shown in Fig. 24. However, if the universe starts to expand at a radius $a_{i} \geq a_{2}$, it will expand forever since $\ddot{a}>0$. A big rip singularity finally develops, since $\rho \rightarrow \infty$ as $a \rightarrow \infty$.

Case C.3.5.2) $|\Lambda|=\left|\Lambda_{c}\right|$ : In this case, there exists a static point $a_{m}$, at which $V\left(a_{m}\right)=$ $V^{\prime}\left(a_{m}\right)=0$, as can be seen in Fig. 23, where $a_{m}=[3(|w|-1) \mathcal{C}]^{-1 /(3|w|-1)}$. Therefore, if the universe starts to expand from $a=0$, it will continue to expand until $a=a_{m}$ with $\ddot{a}<0$. Since $\dot{a}=0=\ddot{a}$ at $a=a_{m}$, the universe will become static at that point. However, it is not stable, and with small perturbations, the universe will either collapse until $a=0$ or expand forever with $\ddot{a}>0$. It should be noted that spacetime is not singular at $a=0$; thus, if it collapses, it begins expanding again when the point $a=0$ is reached. If the universe initially has a radius $a_{i}>a_{m}$, from Fig. 23 it will continue expanding forever, since $V^{\prime}(a)$ is always negative. A big rip singularity will ultimately develop at $a=\infty$.

Case C.3.5.3) $|\Lambda|<\left|\Lambda_{c}\right|$ : In this case, the potential $V(a)$ is negative for all $a$, as shown in Fig. 23. Therefore, the corresponding solutions have no turning points. If the universe initially starts to expand from $a=0$, it will expand forever. However, the potential has a 


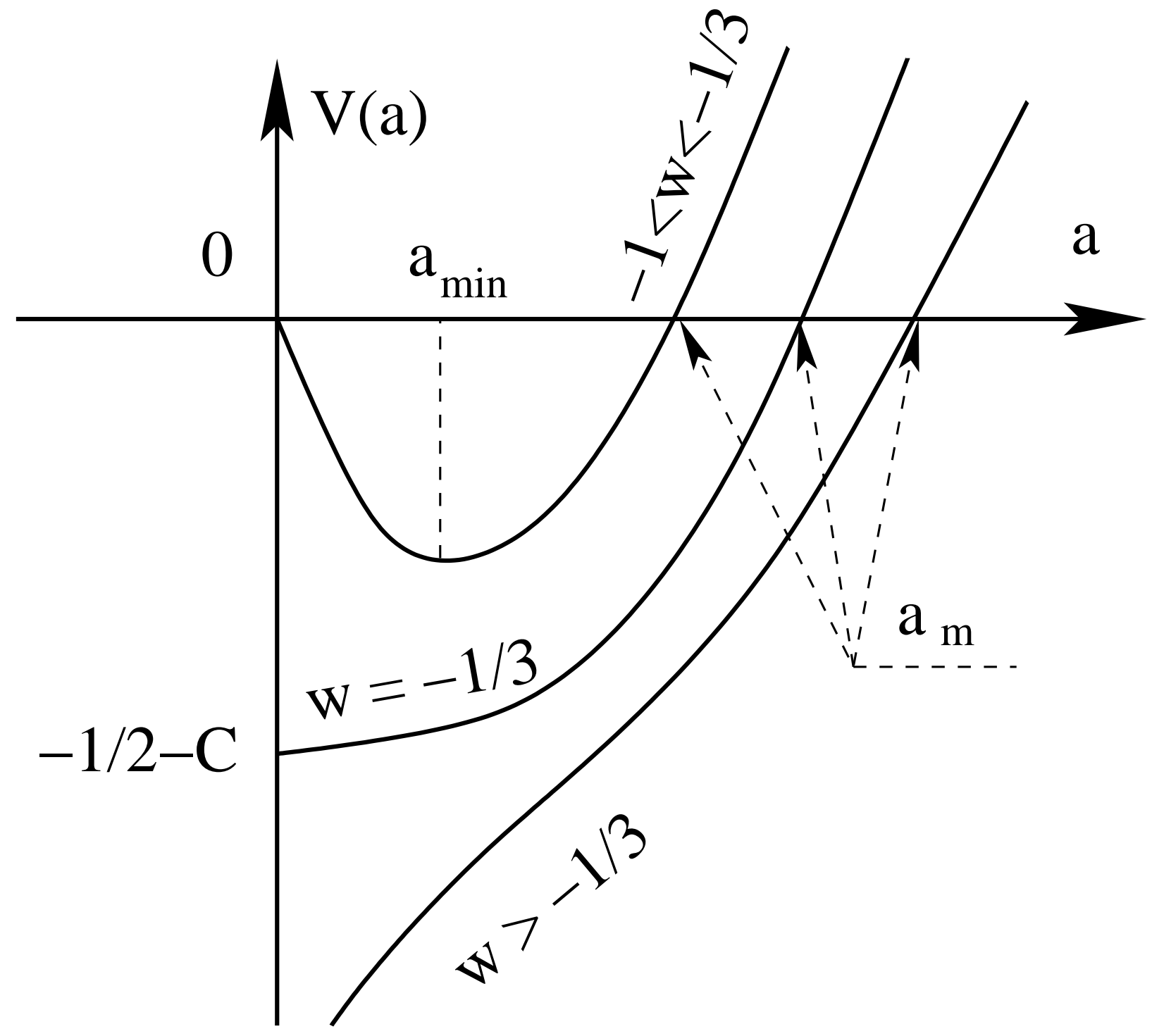

FIG. 19: The potential given by Eq.(2.49) for $k=-1, \Lambda<0$ and $w>-1$.

maximum at $a=a_{m}$, for which

$$
\ddot{a}= \begin{cases}<0, & a<a_{m}, \\ =0, & a=a_{m}, \\ >0, & a>a_{m} .\end{cases}
$$

Thus, the expansion of the universe decelerates initially; however, the expansion begins to accelerate once it reaches $a_{m}$. As in the previous two cases, a big rip singularity develops at $a=\infty$ 


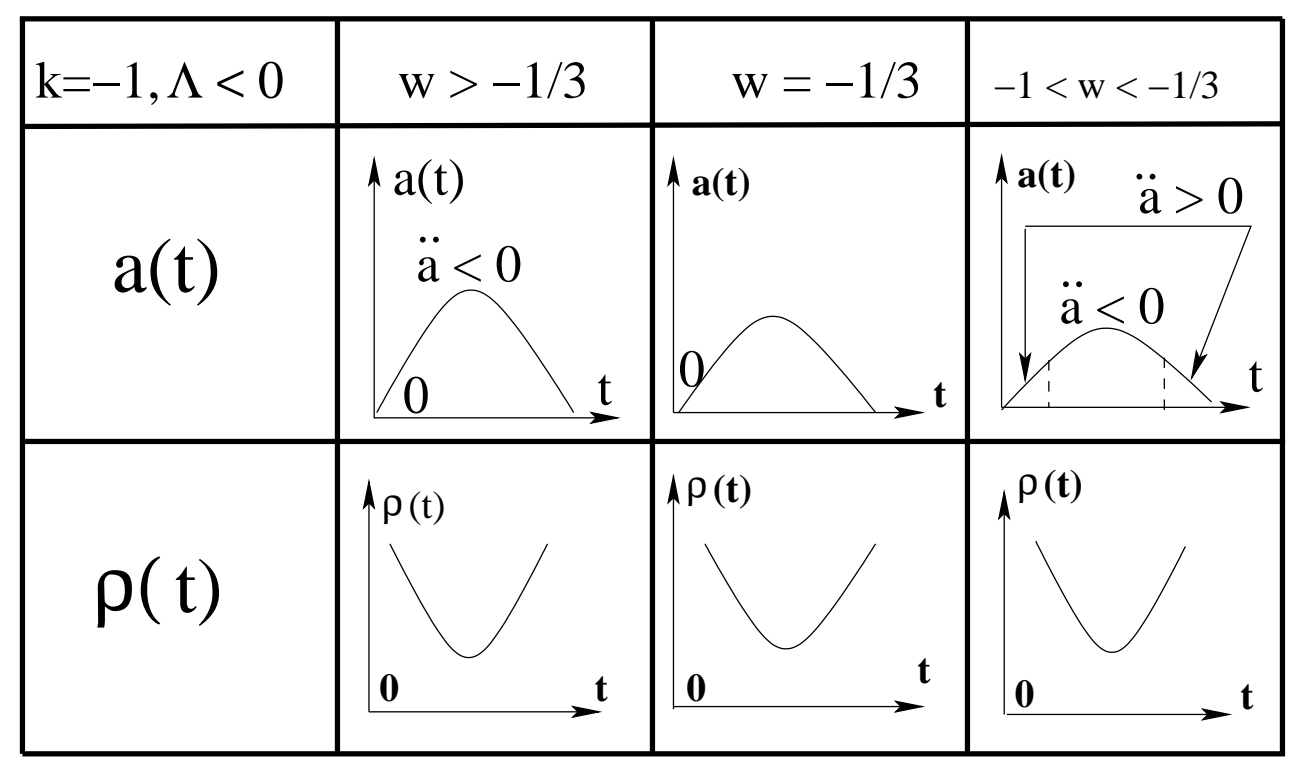

FIG. 20: The expansion factor $a(t)$, the acceleration $\ddot{a}(t)$, and the energy density $\rho(t)$ for $k=$ $-1, \Lambda<0$ and $w>-1$. There are both big bang and big crunch singularities for all cases with $w>-1$.

\section{CONCLUSIONS}

In this paper, we have systematically studied the solutions of the Friedmann-RobertsonWalker (FRW) universe with a cosmological constant and a perfect fluid having the equation of state $p=w \rho$, where $w$ is an arbitrary real constant, and $p$ and $\rho$ denote the pressure and energy density of the fluid, respectively. With the motion of the universe in the form Eq.(1.1), we have been able to classify all of the solutions according to the values of $k, \rho_{0}, w$ and $\Lambda$, by analogy with one-dimensional motion in classical mechanics [13]. These solutions are classified and presented in Figs. 2, 4, 6, 8, 10, 12, 14, 16, 18, 20, 22, and 24, Such classifications are unique, as the potential $V(a)$ depends on these parameters, and different choices of them will correspond to different regions in the phase space, and consequently to different motions of the universe. Some particular cases have previously been discussed in various standard textbooks. In some cases, we have corrected errors in classification and interpretation.

In this paper, we have studied the FRW universe in the Einstein theory of gravity. As pointed out in Introduction, such studies can easily be applied to all models in which the evolution of the universe can be cast in the form of Eq.(1.1] [3], including brane worlds in 


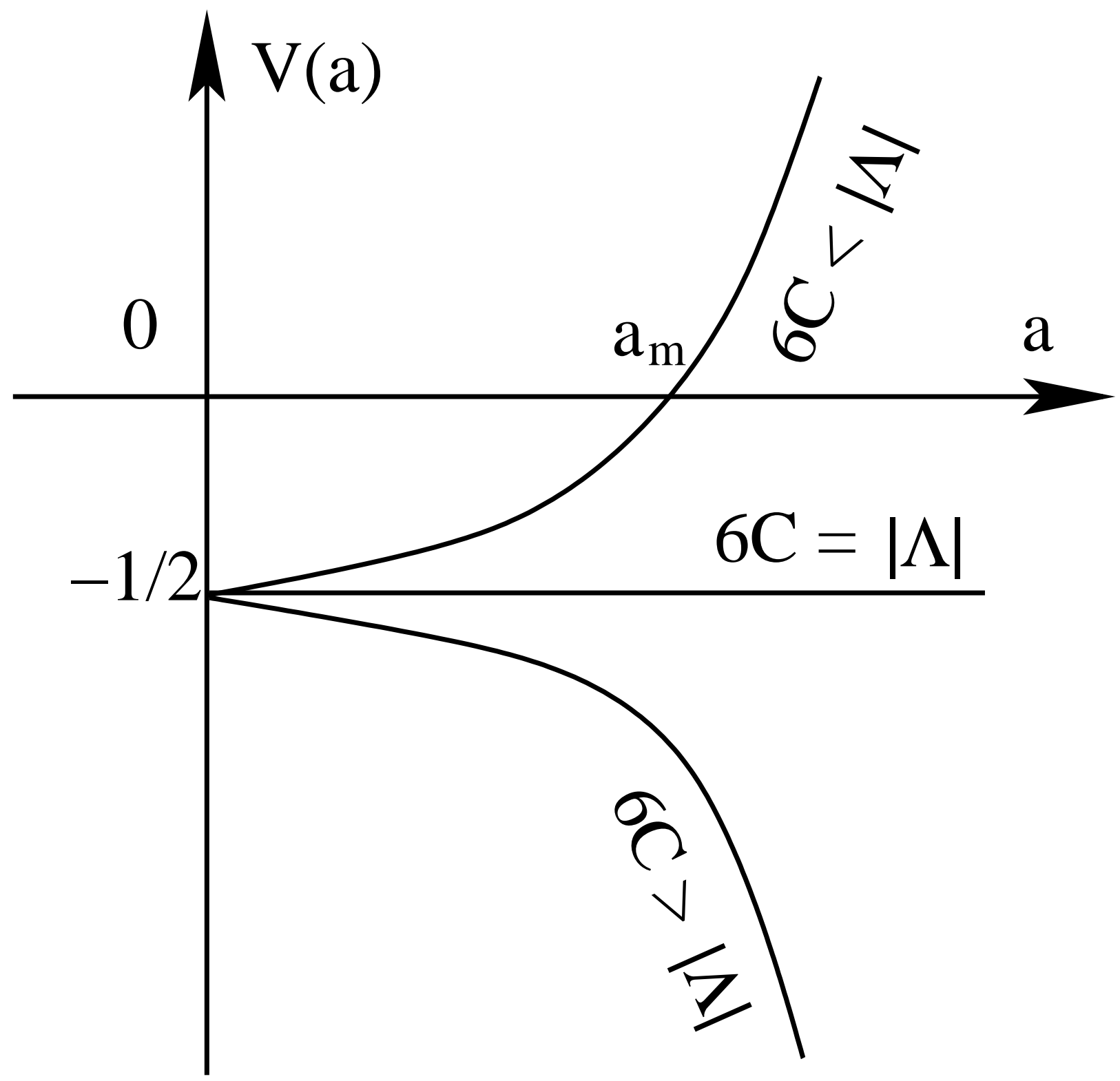

FIG. 21: The potential given by Eq.(2.49) for $k=-1, \Lambda<0$ and $w=-1$.

string/M theory [19], asymmetric branes [20], and cosmological models in the Horava-Lifshitz gravity [21].

\section{Acknowledgments}

We would like very much to express our gratitude to Brandon Bear for his critical reading of this paper and valuable suggestions. AW is supported in part by DOE Grant, DE-FG02- 


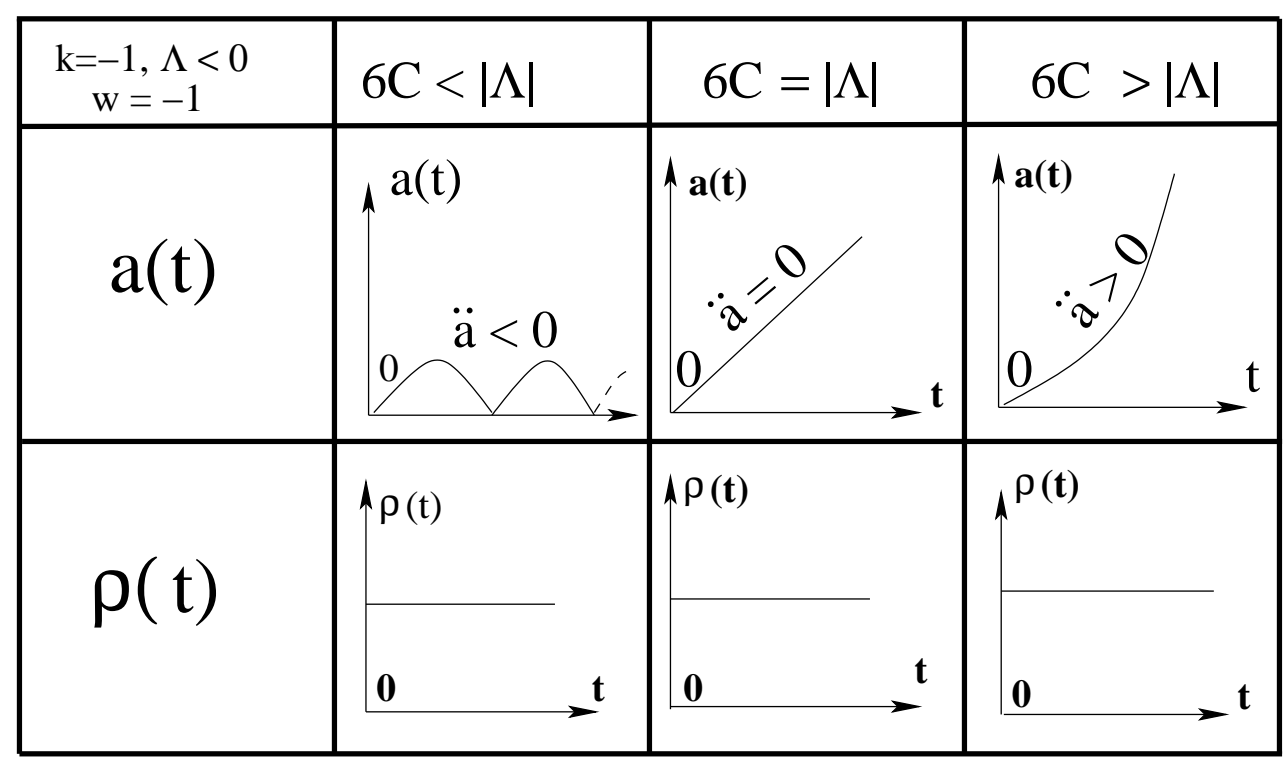

FIG. 22: The expansion factor $a(t)$, the acceleration $\ddot{a}(t)$, and the energy density $\rho(t)$ for $k=$ $-1, \Lambda<0$ and $w=-1$. The spacetime is not singular in any of these cases.

10ER41692

[1] A.G. Riess et al., Astron. J. 116, 1009 (1998); S. Perlmutter et al., Astrophys. J. 517, 565 (1999).

[2] A.G. Riess et al., Astrophys. J. 607, 665 (2004); P. Astier et al., Astron. and Astrophys. 447, 31 (2006); D.N. Spergel et al., astro-ph/0603449; W.M. Wood-Vasey et al., astro-ph/0701041; T.M. Davis et al., astro-ph/0701510.

[3] V. Sahni and A. A. Starobinsky, Int. J. Mod. Phys. D 9, 373 (2000); P.J.E. Peebles and B. Ratra, Rev. Mod. Phys 75, 559 (2003); T. Padmanabhan, Phys. Rep. 380, 235 (2003); V. Sahni, "Dark Matter and Dark Energy," arXiv:astro-ph/0403324 (2004); The Physics of the Early Universe, edited by E. Papantonopoulos (Springer, New York 2005), P. 141; T. Padmanabhan, Proc. of the 29th Int. Cosmic Ray Conf. 10, 47 (2005); E.J. Copeland, M. Sami, and S. Tsujikawa, "Dynamics of dark energy," arXiv:hep-th/0603057 (2006); E.W. Kolb, "Cosmology and the Unexpected," arXiv:0709.3102, E. Linder, "Mapping the cosmological expansion," arXiv:0801.296; J.A. Frieman, M.S. Turner, and D. Huterer, "Dark Energy and the Accelerating Universe," arXiv:0803.0982. 


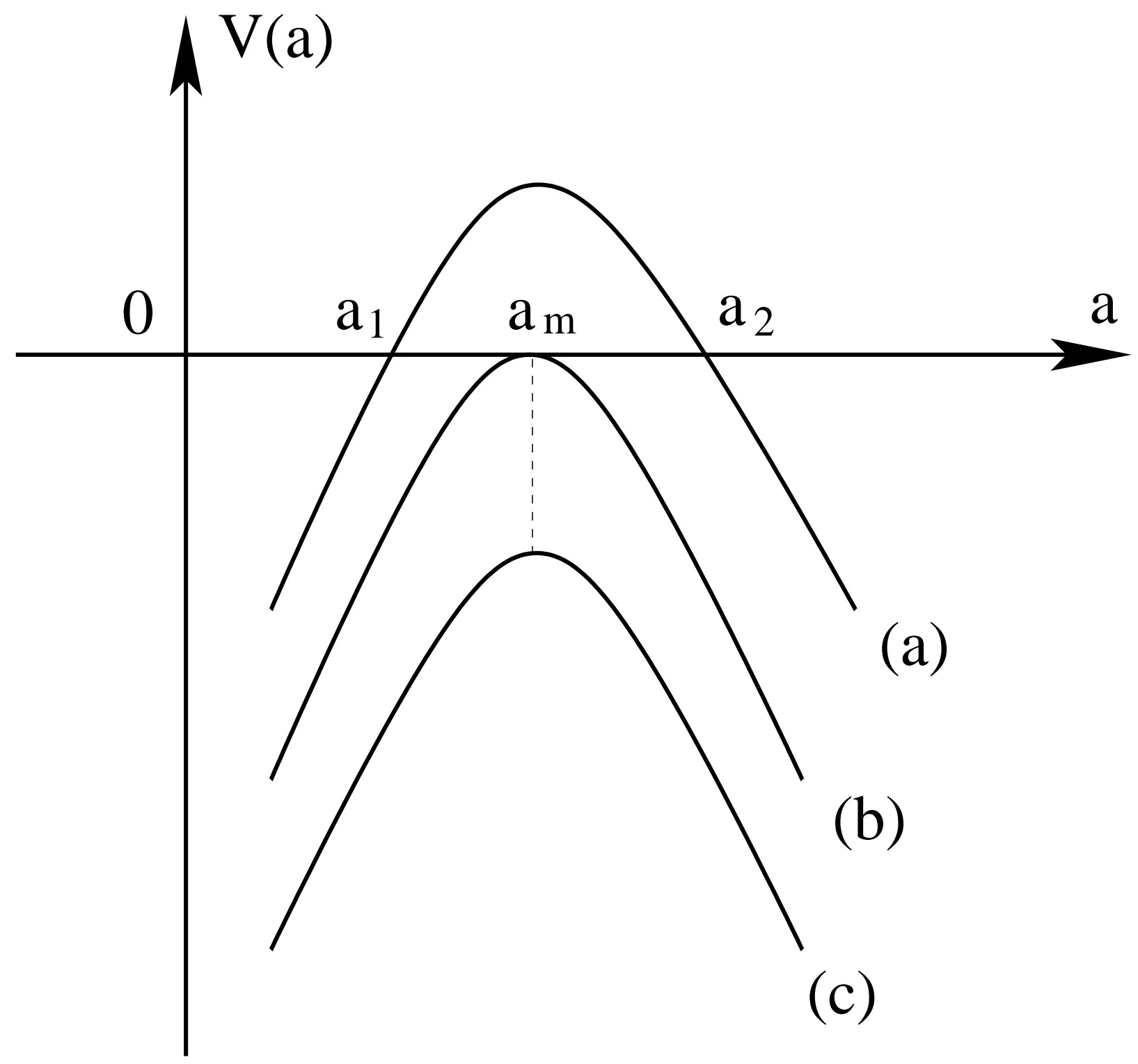

FIG. 23: The potential given by Eq.(2.49) for $k=-1, w<-1$ and $\Lambda<0$, where $\Lambda_{c}$ is given by Eq.(2.50). Curve (a) is for $|\Lambda|>\left|\Lambda_{c}\right|$; (b) is for $|\Lambda|=\left|\Lambda_{c}\right|$; and (c) is for $|\Lambda|<\left|\Lambda_{c}\right|$.

[4] A. Albrecht, et al., "Report of the dark energy task force," arXiv:astro-ph/0609591.

[5] S. Sullivan, A. Cooray, and D.E. Holz, arXiv:0706.3730; A. Mantz, et al., arXiv:0709.4294.

[6] A.D. Chernin, et al., arXiv:0704.2753; arXiv:0706.4171,

[7] S. Weinberg, Rev. Mod. Phys. 61, 1 (1989); S.M. Carroll, arXiv:astro-ph/0004075; T. Padmanabhan, Phys. Rept. 380, 235 (2003); S. Nobbenhuis, arXiv:gr-qc/0411093; J. Polchinski, arXiv:hep-th/0603249, J..M. Cline, arXiv:hep-th/0612129.

[8] W. Fischler, et al., JHEP, 07, 003 (2001); J.M. Cline, ibid., 08, 035 (2001); E. Halyo, ibid., 


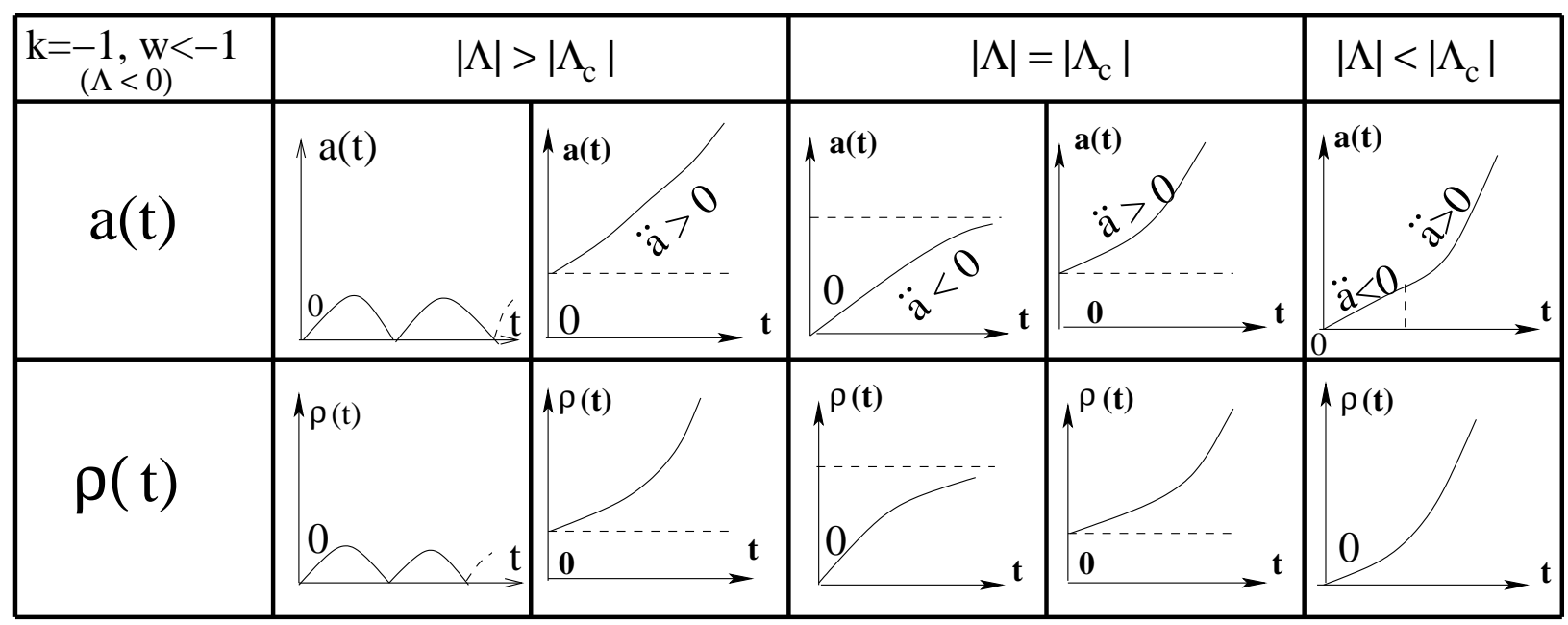

FIG. 24: The expansion factor $a(t)$, the acceleration $\ddot{a}(t)$, and the energy density $\rho(t)$ for $k=$ $-1, w<-1$ and $\Lambda<0$, where $\Lambda_{c}$ is given by Eq.(2.50). There are big rip singularities in all cases, except when $|\Lambda| \geq\left|\Lambda_{c}\right|$.

10, 025 (2001); S. Hellerman, ibid., 06, 003 (2003).

[9] L.M. Krauss and R.J. Scherrer, arXiv:0704.0221; and references therein.

[10] R. R. Caldwell, R. Dave and P. J. Steinhardt, Phys. Rev. Lett. 80, 1582 (1998); A. R. Liddle and R. J. Scherrer, Phys. Rev. D59, 023509 (1999); P. J. Steinhardt, L. M. Wang and I. Zlatev, ibid., 59, 123504 (1999).

[11] G. R. Dvali, G. Gabadadze and M. Porrati, Phys. Lett. B484, 112 (2000); C. Deffayet, ibid., 502, 199 (2001); V. Sahni and Y. Shtanov, JCAP, 0311, 014 (2003).

[12] S. Capozziello, S. Carloni, and A. Troisi, arXiv:astro-ph/0303041; S.M. Carroll, et al, Phys. Rev. D70, 043528 (2003); S. Nojiri and S.D. Odintsov, ibid., 68, 123512 (2003).

[13] H. Goldstein, C. Poole, and J. Safko, Classical Mechanics, Third edition (Addison Wesley, New York, 2002).

[14] M. Szydlowski and W. Czaja, PRD 69, 083507; 083518 (2004); Ann. Phys. 320, 261 (2005); M.Szydlowski, Int. J. Mod. Phys. A 20, 2443 (2005); M. Szydlowski and O.Hrycyna, Gen. Relativ. Grav. 38121 (2006); M. Szydlowski and O.Hrycyna, and A. Krawiec, JCAP, 06, 010 (2007); and M. Szydlowski and O.Hrycyna, J. Math. Phys. 49, 072703 (2008).

[15] T. Chiba, R. Takahashi, and N. Sugiyama, Class. Quantum Grav. 22, 3745 (2005).

[16] R. d'Inverno, Introducing Einstein's Relativity (Clarendon Press, Oxford, 2003).

[17] S. Hawking and G.F.R. Ellis, The Large Scale Structure of Space-Time (Cambridge University 
Press, 1972).

[18] S. Dodelson, Modern Cosmology (Academic Press, New York, 2003).

[19] Y.-G. Gong, A. Wang, and Q. Wu, Phys. Lett. B663, 147 (2008) [arXiv:0711.1597]; and A. Wang and N.O. Santos, Phys. Lett. B 669, 127 (2008) arXiv:0712.3938; arXiv:0808.2055; Q. Wu, N.O. Santos, P. Vo, and A. Wang, JCAP, 09004 (2008) arXiv:0804.0620; Q. Wu, Y. Gong, and A. Wang, JCAP, 06, 015 (2009) [arXiv:0810.5377].

[20] A. Padilla, Class. Quantum Grav. 22, 681 (2005); 22, 1087 (2005); K. Koyama and K. Koyama, Phys. Rev. D72, 043511 (2005); C. Charmousis, R. Gregory, and A. Padilla, arXiv:0706.0857; Y. Shtanov, et al, arXiv:0901.3074; and references therein.

[21] P. Horava, JHEP, 03, 020 (2009); Phys. Rev. D79, 084008 (2009); Phys. Rev. Lett. 102, 161301 (2009); T. takahashi and J. Soda, arXiv:0904.0554; G. Calcagni, arXiv:0904.0829; E. Kiritsis and G. Kofinas, arXiv:0904.1334; H. Lü, J. Mei, and C.N. Pope, arXiv:0904.1595; S. Mukohyama, arXiv:0904.2190; R. Brandenberger, arXiv:0904.2835; A. Volovich and C. Wen, arXiv:0904.2455; R.-G. Cai, Y. Liu, and Y.-W. Sun, arXiv:0904.4104; X. Gao, arXiv:0904.4187; E. Colgain and H. Yavartanoo, arXiv:0904.4357; A. Wang and Y. Wu, JCAP, 07, 012 (2009) arXiv:0905.4117]; A. Wang and R. Maartens, arXiv:0907.1748; and references therein. 